\title{
Inmigración en España: un análisis de las probabilidades de pérdida de empleo (2005-2013)
}

\author{
Eva Medina Moral \\ Universidad Autónoma de Madrid
}

\begin{abstract}
Resumen
La crisis económica ha impactado en España con mayor intensidad sobre los trabajadores inmigrantes que sobre los nativos, de forma que las diferencias en la probabilidad de perder el empleo entre ambos grupos casi se han triplicado durante la recesión. El objetivo de este estudio es determinar si esta tendencia se explica por diferentes características laborales entre ambos colectivos, o si existen otras causas no observables asociadas a una peor valoración de los inmigrantes en el mercado laboral. La metodología aplicada es una extensión de la descomposición de Oaxaca-Blinder (método generalizado de descomposición de diferencias en el primer momento de Yun) válida para modelos logit. Los resultados muestran que las diferencias entre ambos grupos se deben a una mayor concentración de los inmigrantes en segmentos laborales con menores costes de despido (contrato temporal y con poca antigüedad). Aunque el componente discriminatorio no es muy elevado, se observa una tendencia creciente en el mismo durante el periodo de recesión, lo que supone mayores dificultades para la estabilización laboral de este colectivo.
\end{abstract}

Palabras clave: inmigración, probabilidad pérdida de empleo, descomposición OaxacaBlinder, descomposición de Yun.

Clasificación JEL: C25, J61, J64, J71.

\begin{abstract}
The economic crisis has affected more intensively immigrant workers in Spain compared to natives. Differences in the probability of job losing between both groups have almost tripled during the recession. The objective in this paper is to analyze whether this trend can be attributed to differences in workers' characteristics, or alternatively it is originated by some other unobservable variables linked to a lower valuation of immigrants in the labour market. For this purpose, we use an extension of the Oaxaca-Blinder decomposition (decomposing differences in the first moment proposed by Yun), which is valid for logit models. Our results show that the differences between both groups of workers stem from a higher concentration of immigrants in market niches with lower firing costs (temporary contracts with little seniority). Although the discriminatory component is not too high, an increasing trend is observed during the recession, thus creating more difficulties for immigrant workers to achieve job stability.
\end{abstract}

Keywords: immigration, probability of job loss, Oaxaca-Blinder decomposition, Yun decomposition.

JEL classification: C25, J61, J64, J71. 


\section{Introducción. Contextualización del problema}

España ha sido tradicionalmente uno de los países de la Unión Europea con mayor tasa de paro. En la década de los noventa, según datos de EUROSTAT, los parados representaban en torno al 17 por 100 de la población activa, más de 7 puntos porcentuales por encima de la media europea. La preocupación de las autoridades gubernamentales por el empleo, unida al ciclo expansivo que registró la economía española desde mediados de los noventa, permitió un descenso progresivo en la tasa de paro que llegó a registrar un mínimo de 8,3 por 100 en el año 2007. Sin embargo, esta tendencia se ha visto truncada por el inicio de la crisis económica en 2008 que, aunque ha impactado en el mercado laboral de toda Europa, lo ha hecho con más intensidad en países como España y Grecia. Con todo, la tasa de paro en España se situó en 26,4 por 100 en 2013, lo que ha supuesto un incremento de 18 puntos porcentuales desde el inicio de la crisis.

Los datos resultan aún más preocupantes si tenemos en cuenta que la destrucción de empleo durante el periodo de recesión económica no ha afectado igual a todos los colectivos, siendo el de los inmigrantes uno de los más castigados. Si bien la tasa de paro de los extranjeros se situaba por encima de la nativa en torno a 4 puntos porcentuales durante los años previos a la crisis, esta diferencia se ha triplicado en el año 2013, en el que los inmigrantes han registrado tasas de paro por encima del 37 por 100 .

Detrás del aumento de la tasa de paro se encuentra el deterioro de la probabilidad de encontrar un empleo, así como el incremento en la probabilidad de perderlo, observándose nuevamente grandes diferencias ligadas a la nacionalidad. En relación a la probabilidad de encontrar un empleo, ésta ha caído progresivamente desde el 40 por 100, durante el periodo anterior a la crisis, hasta valores por debajo del 15 por 100 en la actualidad. Este hecho ha supuesto un incremento en la duración de los episodios de paro que han pasado de los casi 8 meses en 2005-2008 a unos 13 meses en 2010-2011 (García Serrano et. al., 2012). Atendiendo a la nacionalidad se observa que si bien la probabilidad de encontrar un empleo es superior en los extranjeros en 12 puntos porcentuales durante el periodo de expansión económica, dicha ventaja desaparece durante la crisis, registrándose en la actualidad tasas similares o ligeramente superiores en los nativos.

En relación a la probabilidad de perder el empleo, y según datos de la Encuesta de Población Activa (EPA), esta variable también registra fuertes diferencias entre nativos e inmigrantes que se han intensificado durante el periodo de recesión económica, sobre todo durante los primeros años de la crisis. Tal y como se observa en la Figura 1, la crisis económica ha incrementado la probabilidad de perder el empleo ${ }^{1}$ tanto en nativos (desde un 3,7 por 100 hasta un 6,1 por 100) como en inmigrantes

${ }^{1}$ La probabilidad de perder el empleo se calcula como el porcentaje de personas que estando ocupadas en $t-1$ han pasado en el periodo $\mathrm{t}$ (trimestre siguiente) a ser parados o inactivos desanimados. No se ha tenido en cuenta, por tanto, la transición laboral desde la ocupación a la inactividad por el resto de causas. 
(desde un 6 por 100 hasta un 11,4 por 100). Sin embargo, el impacto ha sido mayor en este segundo colectivo, lo que ha supuesto que la diferencia entre ambos grupos haya crecido un 130 por 100 durante el periodo de recesión (desde 2,3 puntos porcentuales a 5,3 puntos porcentuales). Si el análisis se desagrega por género, se observa que el mayor impacto se ha registrado entre los hombres inmigrantes.

Parte del incremento de esta brecha se justifica por la existencia de diferentes características personales y laborales entre ambos colectivos. Así, los inmigrantes tienen una menor capacidad productiva, derivada de una menor dotación de capital humano, o una mayor concentración en puestos de trabajo más sensibles a los ciclos económicos (ocupaciones de baja cualificación, concentración en el sector de la construcción, mayor presencia de temporalidad, ...) lo que justificaría su mayor exposición a salir del mercado laboral en periodos de recesión económica. Sin embargo, cabe plantarse si más allá de estas causas existen otro tipo de factores inobservables, que pudieran estar relacionados con la existencia de un componente discriminatorio contra el inmigrante.

En este contexto, este estudio pretende aportar evidencia empírica, utilizando una extensión de la metodología de Oaxaca-Blinder (Oaxaca 1973; Blinder 1973) para modelos no lineales, sobre el grado y determinantes de las diferencias detectadas en la probabilidad de perder el empleo entre nativos y extranjeros durante el periodo de crisis económica, tratando de explicar si dichas diferencias están justificadas por la existencia de diferentes características estructurales entre ambos colectivos (personales y laborales) o por el contrario, se deben a que los inmigrantes son peor valorados en el mercado laboral. Este segundo caso podría asociarse a la presencia de un componente discriminatorio en el sentido de que, ante una situación económica desfavorable, el empresario decide despedir, a igualdad de condiciones, al tra-bajador extranjero antes que al nativo.

Este tipo de análisis son importantes tanto desde un punto de vista social como económico, y permiten disponer de evidencia como punto de partida para el desarrollo de políticas sociales y económicas que faciliten la integración de los inmigrantes, reduciendo las diferencias entre extranjeros y nativos y generando una sociedad más equitativa. En este sentido, las conclusiones permiten detectar que, si bien la cuantía de dicho componente discriminatorio es baja, se ha registrado una tendencia creciente durante el periodo de recesión, y en concreto en la valoración que el empresario hace de los trabajadores inmigrantes con contrato temporal y poca antigüedad en la empresa, quienes sufren más despidos que los nativos con las mismas características. Aún con todo, la principal causa que explica la diferencia en la probabilidad de perder el empleo de inmigrantes y nativos, y el incremento de la misma durante el periodo de recesión, es la existencia de diferencias en las características laborales de ambos colectivos, asociadas principalmente a la mayor concentración de los trabajadores inmigrantes en los segmentos con menores costes de despido (contratos temporales y con poca antigüedad en la empresa). 


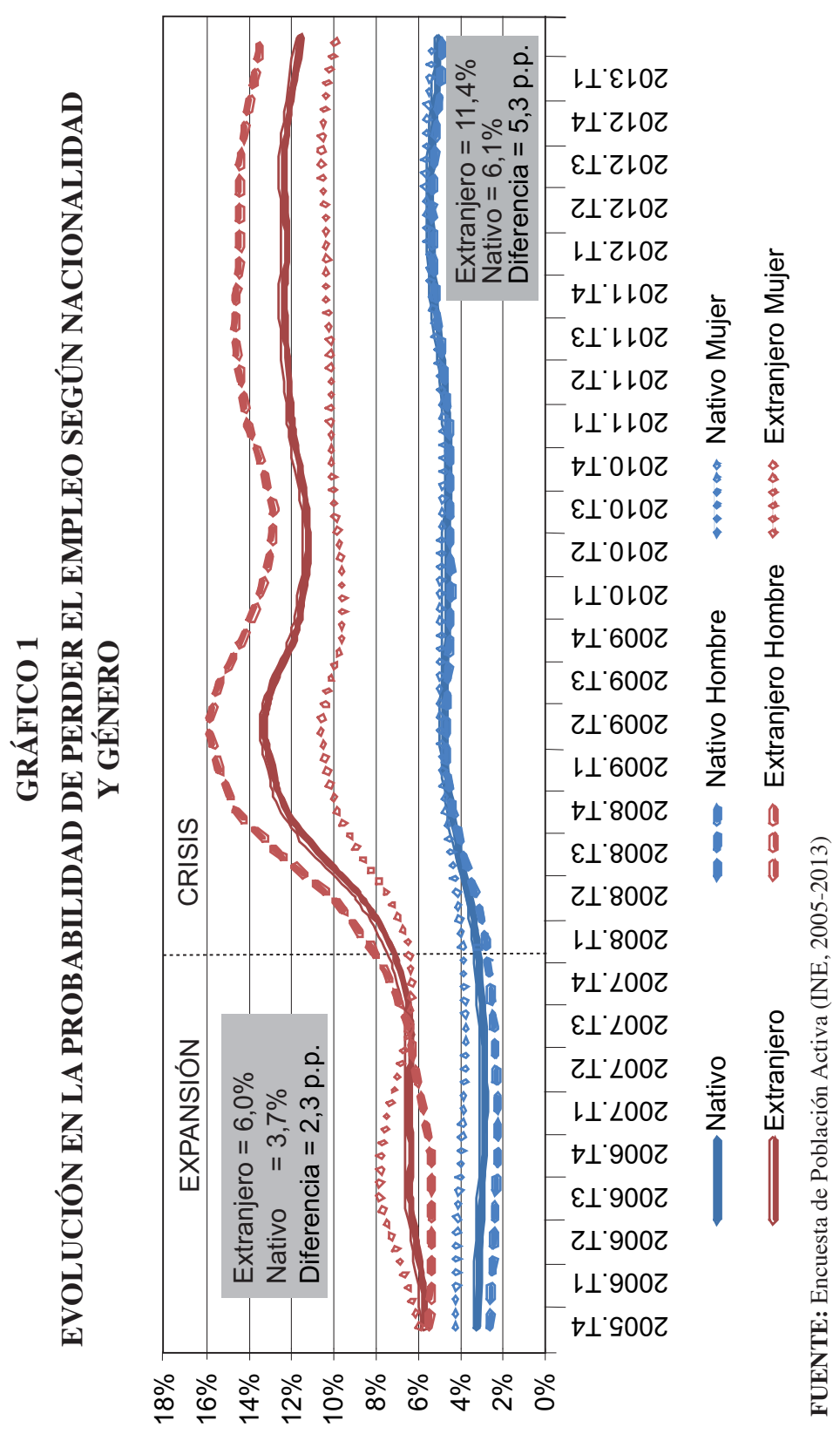


El estudio se estructura en los siguientes apartados: revisión bibliográfica de la materia, descripción de los datos utilizados en el análisis, desarrollo de la metodología empleada y detalle de los resultados obtenidos. La investigación se cierra con un resumen de las principales conclusiones.

\section{Revisión bibliográfica: literatura sobre inmigración y mercado laboral}

Las investigaciones académicas en relación a la migración se han centrado en analizar los determinantes de la migración y de su retorno, el desarrollo de políticas migratorias, y la relación entre los inmigrantes y el mercado laboral del país receptor.

En esta última línea de trabajo, algunos estudios han tratado de cuantificar el impacto de la llegada de inmigrantes en el empleo nativo. Los trabajos en este sentido se han centrado en contrastar la existencia de una relación negativa entre la tasa de ocupación y los salarios de los nativos, y el número (o proporción) de inmigrantes extranjeros. Destacan los trabajos de Friedberg y Hunt (1995), Borjas et al. (1997) y Borjas (1999), en Estados Unidos; Greenwood y McDowell (1986), De New y Zimmermann (1994) y Pischke y Velling (1997) en Europa; y los de Dolado et. al. (1997), Herrarte et. al. (2007), Carrasco et. al. (2008) y Amuedo Dorantes y De la Rica (2013) para el caso español. En todos los casos se concluye que o bien la inmigración no tiene efectos, o bien que éstos son de escasa magnitud.

Otra corriente de estudios han tratado de analizar los procesos de asimilación del inmigrante en el mercado laboral del país receptor. En este sentido se trata de contrastar si las diferencias laborales inicialmente detectadas entre nativos e inmigrantes se reducen a medida que se incrementa el tiempo de permanencia del inmigrante. La mayor parte de la literatura empírica corrobora la hipótesis de asimilación del inmigrante tanto en términos de salario (Chiswick (1978), Borjas (1995), Friedberg (2000), Duleep y Dowhan (2002), Constant y Massey (2003) y Card (2005)), como en términos de la capacidad de un inmigrante para encontrar un trabajo (Chiswick et al. (1997), Wheatly (1999), Bevelander y Nielsen (2001) y Clark y Lindley (2005)).

En el caso de España, el primer estudio que analiza la asimilación de los inmigrantes en el mercado laboral es el de Amuedo Dorantes y de la Rica (2007), en el que, con datos del Censo de Población (INE, 2001) y de la Encuesta de Estructura Salarial (INE, 2002), demuestran que la asimilación en el mercado laboral de los inmigrantes varía por género, origen y educación, siendo ésta más rápida entre los hombres más cualificados. El trabajo posterior de Fernández y Ortega (2008), con datos de la EPA (INE, 1996-2005), muestra un claro éxito en la asimilación del inmigrante, ya que 5 años después de su llegada la probabilidad de ser desempleado es incluso más baja que la de los nativos, aunque la calidad de los trabajos obtenidos es peor, concentrándose en empleos temporales para los cuales están sobrecualificados. 
Sin embargo, en el caso español, la llegada de inmigrantes presenta características específicas que la diferencia de otros países, ya que ésta se ha producido de manera muy intensa en un periodo de tiempo muy escaso. Así, mientras que en el año 2000, la población inmigrante representaba tan sólo el 3 por 100 de la población activa, esta cifra se situó en el 16 por 100 en el año 2009. Si bien la crisis económica ha supuesto un freno a esta tendencia, los niveles siguen siendo elevados en la actualidad (14 por 100 en 2013). En este sentido, parte de la literatura sobre el tema ha tratado de analizar si la asimilación de este fuerte crecimiento de los trabajadores inmigrantes se ha producido en las mismas condiciones que las de los trabajadores nativos o si, por el contrario, el tan rápido e intenso proceso de asimilación ha generado situaciones de discriminación negativa para el colectivo inmigrante.

Pocos estudios sostienen que una parte de las diferencias salariales se explica por la existencia de discriminación, en línea con las conclusiones obtenidas en Simón et. al. (2008). Los autores, con datos de la Encuesta de Estructura Salarial (INE, 2002), encuentran que las diferencias en la distribución salarial entre nativos e inmigrantes vienen explicadas por la existencia de diferentes características laborales, con importante influencia del lugar de trabajo y la segregación ocupacional. Sin embargo, Canal Domínguez y Rodríguez Guitiérrez (2008), con los mismos datos de la Encuesta de Estructura Salarial (INE, 2002), encuentran indicios de discriminación negativa contra el salario del inmigrante, aunque sólo en el segmento de salarios bajos y en mayor medida en el caso de la mujer.

No obstante, la mayoría de los estudios se centran en la búsqueda de discriminación en los salarios, obviando otras variables, más relacionadas con los procesos de contratación, donde pudiera ser más fácil generar situaciones discriminatorias. En este sentido, Caparrós y Navarro (2010), con datos de la Encuesta de Población Activa (INE, 2005-2009), analizan la diferencia existente en la contratación indefinida de nativos e inmigrantes, concluyendo que un porcentaje elevado de la misma no viene explicado por las diferencias en las características de los trabajadores, sino por el hecho de que los factores que influyen sobre la contratación indefinida son valorados de manera distinta por el empresario según se trate de nativos o inmigrantes.

Por otro lado, ninguno de los estudios analiza el impacto que la crisis económica está teniendo en los procesos de asimilación, ya que los periodos analizados no incluyen información sobre los años de recesión. En este contexto, la presente investigación pretende aportar evidencia empírica sobre si la crisis actual está suponiendo un freno a los procesos de asimilación, haciendo aflorar situaciones discriminatorias que dificultan el acceso del colectivo inmigrante al mercado laboral. Para ello trataremos de analizar si el incremento que ha registrado, durante el periodo de crisis, la diferencia existente entre la probabilidad de perder el empleo de inmigrantes y nativos se puede explicar por una mayor concentración de los primeros en los segmentos laborales más sensibles al ciclo económico, o si por el contrario existen otras causas que pudieran asimilarse a la existencia de un componente discriminatorio. 


\section{Descripción de los datos. EPA-flujos (2005-2013)}

Para poder analizar la transición laboral de los individuos desde la situación de empleo a desempleo, es necesario usar datos longitudinales que permitan observar la evolución de un mismo individuo en el tiempo.

Las estadísticas españolas que permiten realizar análisis longitudinales son la Muestra Continua de Vidas Laborales (MCVL) y la EPA flujos, si bien en este estudio se ha utilizado la segunda. Ambas estadísticas presentan ventajas y desventajas. En la MCVL, que cruza estadísticas de la Seguridad Social, Padrón Municipal y Agencia Tributaria, los datos están disponibles para los investigadores más tarde que en la EPA, y sólo recoge información de individuos que cotizan o han cotizado a la Seguridad Social, por lo que no tiene en cuenta la existencia de economía sumergida. A su vez la EPA flujos dispone de un número menor de observaciones que la MCVL y sólo permite realizar el seguimiento de los individuos durante seis trimestres consecutivos. Sin embargo, estas limitaciones de la EPA flujos no afectan al análisis que se presenta en este estudio, en el sentido de que el seguimiento de los individuos se realiza entre dos trimestres consecutivos y los datos incluidos (36 secciones cruzadas comprendidas entre el primer trimestre de $2005^{2}$ y el cuarto trimestre de 2013) constituyen un tamaño muestral suficiente como para garantizar los resultados estadísticos del análisis.

Por otro lado, si bien la Encuesta de Población Activa (EPA) dispone de información detallada para la variable nacionalidad, en los flujos de esta encuesta se reduce el nivel de desagregación con el que se muestra esta variable para garantizar el anonimato de los encuestados, distinguiendo sólo entre individuos con nacionalidad española o extranjera. Esto tiene como inconveniente no poder diferenciarla inmigración económica (procedente de países poco desarrollados) de la no económica con características laborales, esta última, más similares a los nativos. Si bien los inmigrantes económicos representan en torno al 85 por 100 del total de inmigración, es necesario tener en cuenta que los resultados de este estudio se encontrarán ligeramente infravalorados por este hecho.

En el Cuadro 1 se muestra la información de las variables que serán utilizadas en el análisis ${ }^{3}$ : la variable de interés, probabilidad de perder el empleo (cuatro últimas columnas del cuadro); y las variables que explican el hecho de que se produzca la transición desde una situación de empleo a desempleo (filas del cuadro). Estas últimas se relacionan con características personales del individuo (sexo, edad, nivel de formación y estado civil) y laborales asociadas al puesto de trabajo (sector de actividad, antigüedad en la empresa, tipo de contrato y

\footnotetext{
2 No se pueden incluir periodos anteriores al año 2005, ya que en esta fecha el INE modificó la metodología de la EPA incluyendo, entre otras mejoras, una mayor representatividad muestral de la población extranjera.

${ }^{3}$ Sólo se han analizado aquellas personas con edad comprendida entre 16 y 59 años que en el periodo $t-1$ estuvieran ocupadas como asalariados del sector privado. Además se han excluido del análisis las ciudades autónomas de Ceuta y Melilla por tener una muestra poco representativa.
} 
ocupación $\left.{ }^{4}\right)$. Los datos de la tabla muestran, para cada segmento de la población, la distribución de los ocupados según nacionalidad y la probabilidad de perder el empleo según nacionalidad y según momento del ciclo económico. Con todo, los datos nos permiten analizar si los inmigrantes se concentran en aquellos segmentos de la población con mayor probabilidad de perder el empleo y en los que el impacto de la crisis económica ha sido mayor.

Atendiendo a las características de la muestra se observa que los inmigrantes tienen una edad inferior a la de los nativos (se concentran en mayor medida en edades por debajo de los 45 años), un menor nivel educativo (el porcentaje de población con estudios terciarios es 10 puntos porcentuales inferior en el caso de los inmigrantes), una elevada presencia, superior a la nativa, en el sector de la construcción y de la agricultura, una muy superior tasa de temporalidad (mientras que la temporalidad entre los nativos supone el 25 por 100, esta cifra se eleva hasta casi el 50 por 100 en el caso de los inmigrantes), unida a una menor antigüedad en la empresa, y una mayor concentración en las ocupaciones de baja cualificación (el 64 por 100 de la población inmigrante se concentra en este segmento).

Las características individuales que se traducen en mayores tasas de pérdida de empleo son compartidas tanto por españoles como por extranjeros. Es el caso de las mujeres, los jóvenes, los que poseen menor capital humano, tanto en términos de formación como de experiencia laboral, solteros, con contratos temporales y poca antigüedad en la empresa, y que trabajan en el sector agrario en ocupaciones de baja cualificación. Estos son, a su vez, los segmentos de la población donde la crisis económica ha tenido un mayor impacto. Así, los mayores incrementos en la probabilidad de perder el empleo, por encima de 7 puntos porcentuales, se han registrado entre los jóvenes, en el sector agrario y de la construcción, con contratos temporales y baja antigüedad en la empresa.

${ }^{4}$ La variable se agrupó en tres categorías donde alta cualificación incluye dirección de empresas, técnicos y profesionales científicos e intelectuales, técnicos y profesionales de apoyo; media cualificación incluye empleados de tipo administrativo, trabajadores de servicios de restauración, personales y comercio, y trabajadores cualificados en la agricultura y en la pesca; y baja cualificación incluye trabajadores en manufacturas, construcción y minería, operadores de instalaciones y maquinaria y trabajadores no cualificados. 


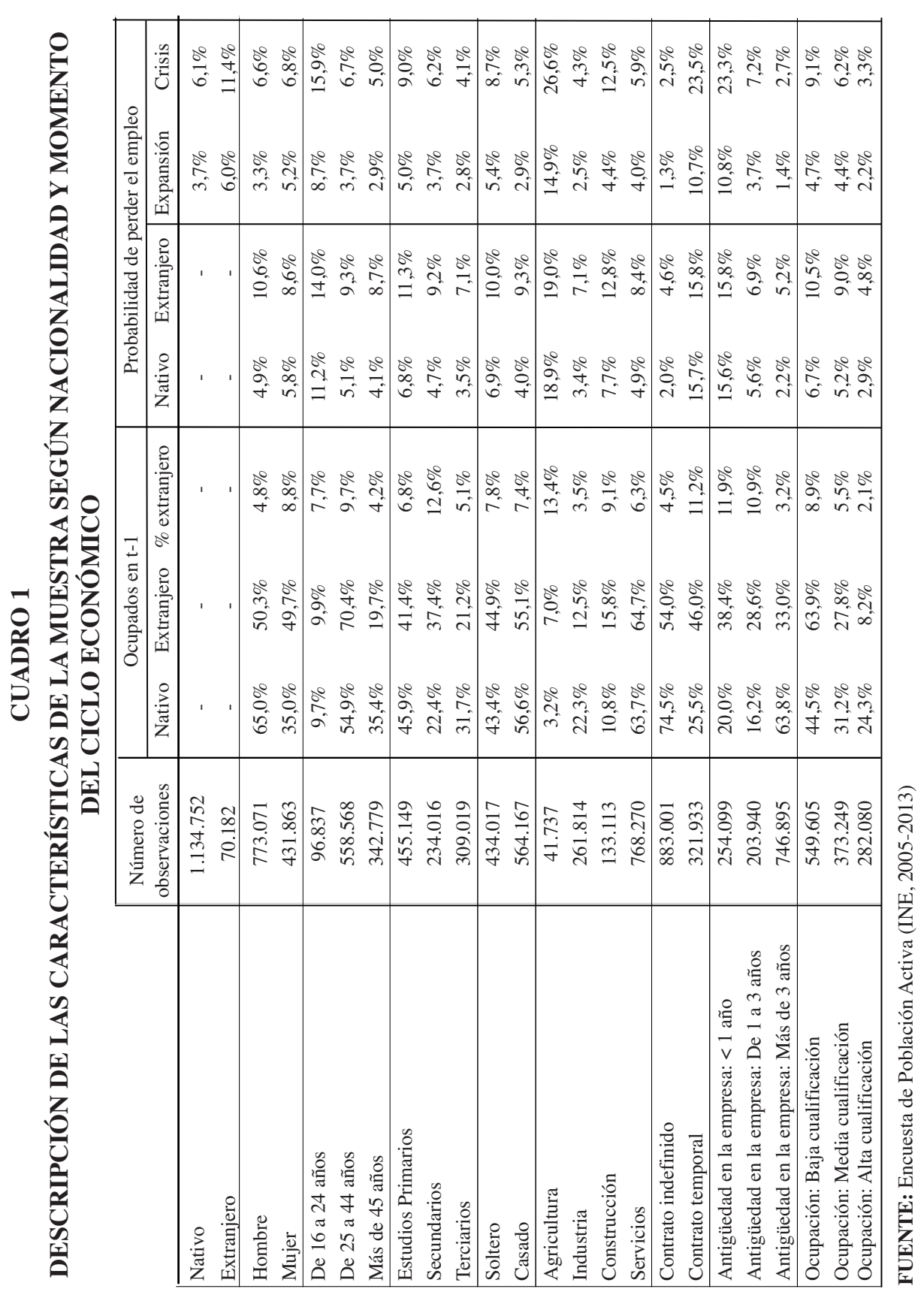


Con todo, el ocupado extranjero se caracteriza, frente al nativo, por mayor presencia en los segmentos más propensos al despido y, a su vez, que más se han visto castigados por la crisis: jóvenes con baja formación, en el sector agrario y de la construcción, con contratos temporales y baja antigüedad en la empresa ocupando puestos de poca cualificación.

Estas diferencias justificarían el hecho de que la probabilidad de perder el empleo sea superior en la población inmigrante que en la nativa, y que, además, esa brecha se haya incrementado con el tiempo, a medida que la crisis ha intensificado sus efectos. En este punto cabría preguntarse si, una vez identificadas las variables que justifican la mayor pérdida de empleo entre los inmigrantes, y controlando el efecto de las mismas sobre dicha probabilidad, es posible aún identificar una mayor propensión a perder el empleo entre la población inmigrante. Es decir, si ante igualdad de condiciones el empresario es más proclive a despedir al inmigrante que al nativo, lo que estaría indicando la presencia de factores no observables asociados a comportamientos discriminatorios atendiendo a la nacionalidad. A esta cuestión tratamos de dar respuesta en los siguientes epígrafes.

\section{Metodología de análisis. Modelo logit y método generalizado de descomposición de diferencias en el primer momento de Yun}

Con el objetivo de analizar si la mayor transición hacia el paro que registra actualmente la población extranjera frente a la española se explica por la existencia de diferencias en las características laborales de ambos colectivos o, si además, existe algún otro factor diferencial, se aplican dos metodologías alternativas. La primera consiste en la estimación econométrica de un modelo de respuesta binaria con ajuste logístico (modelo logit), siguiendo la metodología aplicada en Medina et.al. (2010).

La especificación econométrica utilizada para cuantificar como influye la nacionalidad en la probabilidad de perder el empleo es la correspondiente a un modelo logit según la cual:

$$
P\left(Y_{i}=1\right)=\frac{\exp \left(\beta^{\prime} X_{i}\right)}{1+\exp \left(\beta^{\prime} X_{i}\right)}
$$

Donde $Y_{i}=1$ si el trabajador perdió el empleo y 0 si no lo perdió; $X_{i}$ es un vector que recoge los valores de las variables explicativas para cada individuo y $\beta$ es el vector de los parámetros.

La variable endógena, en todos los casos, se construye comparando la situación laboral de los individuos entre dos trimestres consecutivos. Así, para modelizar la probabilidad de perder el empleo se distingue entre los ocupados en $t-1$ que mantienen su empleo en $\mathrm{t}$ (valor 0 ) y los que lo perdieron (valor 1). 
Las variables utilizadas para explicar la probabilidad de perder el empleo se clasifican en 3 grupos: 1) Características personales del individuo: sexo, edad, nivel formativo, estado civil y nacionalidad, 2) Características laborales: sector de actividad (4 sectores), tipo de contrato (indefinido y temporal), antigüedad en la empresa, y la ocupación (3 categorías) y 3) Ciclo económico, incluyéndose una variable dicotómica que toma el valor 1 si la transición laboral corresponde a un periodo de crisis y 0 en el resto de los casos (se considera el comienzo de la crisis el año 2008). Todas las regresiones incluyen además controles para cada comunidad autónoma, con el fin de considerar efectos específicos de los mercados laborales regionales, y para el trimestre de análisis, controlando con ello el efecto estacional que presenta la probabilidad de pérdida de empleo.

Se plantean tres especificaciones alternativas incorporando sucesivamente en cada una de ellas la variable nacionalidad (que mide si el individuo es extranjero o no), crisis (que mide si la observación pertenece a un periodo de crisis o de expansión) y la interacción entre ambas. Así, en la primera especificación, un parámetro significativo para la variable nacionalidad con signo positivo indicaría que, una vez controlado el efecto del resto de las variables que influyen en la pérdida de empleo, dicha probabilidad sigue siendo mayor en los extranjeros. Es decir, que existen otros factores no observables asociados a la nacionalidad que explican las diferencias. En la segunda especificación se incluye además de la nacionalidad como variable explicativa, la variable crisis. El objetivo es detectar si una vez controlado el impacto que la crisis económica ha tenido en la probabilidad de perder el empleo, el parámetro de la variable que mide la nacionalidad se ve modificado. Por último se incluye, junto a las dos variables anteriores (efectos principales), la interacción entre ambas, para detectar si las diferencias cuantificadas con los modelos anteriores entre nativos y extranjeros se han mantenido constantes antes y después de la crisis. Con todo, un resultado estadísticamente significativo en el parámetro que acompaña a la interacción estará indicando que la crisis afecta de manera distinta al empelo de nativos y extranjeros.

El análisis se realiza para la muestra total y desagregando la misma en hombres y mujeres, debido a las especificidades propias por género del mercado laboral. Se excluyeron del análisis las observaciones pertenecientes al año 2008, por tratarse de un año de transición en el cambio de ciclo económico.

Con esta metodología, si bien el parámetro de la interacción permite cuantificar si durante el periodo de crisis han existido diferencias en la probabilidad de perder empleo según nacionalidad, los efectos del resto de variables sobre la probabilidad de perder el empleo se mantienen constantes en ambos colectivos, inmigrantes y nativos, siendo ésta una restricción que puede resultar no realista. La estimación de muestras separadas según nacionalidad permite resolver este problema. Obtener parámetros distintos en la estimación de los nativos frente a la de los inmigrantes estaría reflejando la existencia de un componente discriminatorio hacia uno de los colectivos, en el sentido de que indicaría que la valoración que hace el mercado laboral sobre el efecto de cada variable en la probabilidad de perder el empleo es distinta según la nacionalidad de procedencia. 
A partir de los resultados obtenidos en dichas estimaciones, la metodología de Oaxaca-Blinder (Oaxaca 1973; Blinder 1973) permite cuantificar qué parte de las diferencias obtenidas en la probabilidad de perder el empleo se deben a características laborales distintas entre los trabajadores nacionales y extranjeros, o a la existencia de diferencias en las repercusiones que dichas características tienen sobre la propensión a perder el empleo entre ambos colectivos. Este segundo componente es el asociado a la existencia de factores inobservables entre los que podría encontrarse un componente discriminatorio. Sin embargo, estos resultados deben ser interpretados con cautela en la medida en que esas diferencias recogen también la existencia de variables omitidas en la especificación por su dificultad de medición (menores niveles de productividad asociadas a las diferencias en la calidad de la educación en los países de origen, diferencias en absentismo laboral, o riesgo de retorno al país de origen, entre otras).

En esta investigación se utiliza una extensión del enfoque tradicional de OaxacaBlinder para el caso de variables endógenas dicotómicas. Concretamente se aplica la descomposición de Yun (Yun, 2004) para modelos no lineales. Partiendo de las probabilidades medias predichas de perder el empleo $\operatorname{Prob}\left(Y_{i}=1\right)_{G}$, donde el subíndice $G$ es igual a $N$ para el colectivo nativo e $I$ para el inmigrante, la diferencia de la probabilidad media se puede expresar en términos de los valores medios de la función de distribución acumulada de la logística $F\left(X_{G} \hat{\beta}_{G}\right)$ como:

$$
\overline{\operatorname{Prob}\left(Y_{\imath}=1\right)_{N}}-\overline{\operatorname{Prob}\left(Y_{\imath}=1\right)_{I}}=\overline{F\left(X_{N} \hat{\beta}_{N}\right)}-\overline{F\left(X_{I} \hat{\beta}_{I}\right)}
$$

La diferencia expresada en (2) se puede descomponer en la suma de dos términos:

$\overline{\operatorname{Prob}\left(Y_{\imath}=1\right)_{N}}-\overline{\operatorname{Prob}\left(Y_{\imath}=1\right)_{I}}=\left(\overline{F\left(X_{N} \hat{\beta}_{N}\right)}-\overline{F\left(X_{I} \hat{\beta}_{N}\right)}\right)+\left(\overline{F\left(X_{I} \hat{\beta}_{N}\right)}-\overline{F\left(X_{I} \hat{\beta}_{I}\right)}\right)$

Donde el primer término recoge las diferencias debidas a las distintas características que definen a los dos grupos de trabajadores, nativos e inmigrantes (diferencia en las características), mientras que el segundo cuantifica la parte debida a diferencias en la valoración que hace el mercado de las características de los dos colectivos analizados para ser expulsados del mercado laboral (diferencia en los coeficientes).

Para determinar la contribución de cada una de las variables explicativas a los dos efectos anteriores,Yun propone una metodología de descomposición conocida como el método generalizado de descomposición de diferencias en el primer momento. Dicha metodología es válida para cualquier forma funcional especificada para la variable dependiente, siempre que dicha variable dependiente sea una función de una combinación lineal de las variables independientes y que dicha función sea diferenciable, restricciones ambas que cumplen los modelos logit. Esta metodología de descomposición consta de dos etapas: en la primera se aproxima la media muestral a través de la función evaluada en la media muestral de las características; mientras que en la segunda etapa se aplica una extensión de Taylor de primer orden para 
linealizar los efectos correspondientes en características y en rendimientos alrededor de los valores medios de cada grupo respectivamente, obteniéndose la siguiente expresión:

$$
\begin{aligned}
& \overline{\operatorname{Prob}\left(Y_{I}=1\right)_{N}}-\overline{\operatorname{Prob}\left(Y_{I}=1\right)_{I}}=\sum_{j=1}^{k} W_{\Delta X}^{j}\left(\overline{F\left(X_{N} \hat{\beta}_{N}\right)}-\overline{F\left(X_{I} \hat{\beta}_{N}\right)}\right)+ \\
& +\sum_{j=1}^{k} W_{\Delta \beta}^{j}\left(\overline{F\left(X_{I} \hat{\beta}_{N}\right)}-\overline{F\left(X_{I} \hat{\beta}_{I}\right)}\right)
\end{aligned}
$$

Donde los pesos de cada variable $j$ sobre las diferencias en características y en coeficientes son $W_{\Delta X}^{j}=\left(\bar{X}_{N}^{j}-\bar{X}_{I}^{j}\right) \hat{\beta}_{N}^{j} /\left(\bar{X}_{N}-\bar{X}_{I}\right) \hat{\beta}_{N}$ y $W_{\Delta \beta}^{j}=\bar{X}_{I}^{j}\left(\beta_{N}^{j}-\hat{\beta}_{I}^{j}\right) / \bar{X}_{I}\left(\hat{\beta}_{N}-\hat{\beta}_{I}\right)$, respectivamente, $\mathrm{y}$ permiten distribuir la contribución de las diferencias totales en características y en coeficientes entre las distintas variables observadas. Dichos pesos cumplen la condición de que $\sum_{j}^{k} W_{\Delta X}^{i}=\sum_{j}^{k} W_{\Delta \beta}^{i}=1$.

Esta metodología tiene la limitación conocida en la literatura económica como el problema de identificación, según el cual la contribución de las variables ficticias a la diferencia de probabilidad media no es robusta a la categoría de referencia elegida. Para evitar este problema se ha aplicado la corrección de normalización propuesta por Gardeazábal y Ugidos (2004), según la cual las ponderaciones se obtienen por mínimos cuadrados restringidos, imponiendo la restricción de identificación consistente en que la suma de los coeficientes de cada conjunto de variables ficticias es igual a cero.

Con todo, la descomposición permitirá, no sólo determinar el origen de la diferencia por nacionalidad en la probabilidad de perder el empleo antes y después de la crisis, sino que además permite identificar que variables contribuyen en mayor medida a dicha diferencia.

\section{Principales resultados}

\subsection{Probabilidad de perder el empleo}

En los Cuadros 2 a 4, se resumen los resultados de las estimaciones logit planteadas para la muestra total, la de hombres y la de mujeres. Todos los parámetros de las variables utilizadas en el análisis obtuvieron resultados estadísticamente significativos y con los signos esperados. Los estadísticos de bondad conjunta se encuentran dentro de los límites aceptables, siendo la muestra de hombres la que registra un mejor ajuste.

En relación a las variables relacionadas con las características personales del individuo, y centrándonos en el modelo más completo (Modelo 3), se observa que la mujer tiene una mayor probabilidad de perder el empleo que el hombre $(0,8$ puntos porcentuales por encima). El tramo de edad en el que se registra una menor propensión a perder el empleo es el comprendido entre 25 y 44 años, con una probabilidad de perder el empleo 0,26 puntos porcentuales inferior para un trabajador de esta edad 
que para otro entre 16 y 24 años. En el caso de los hombres llama la atención que la probabilidad de perder el empleo entre los trabajadores con más de 45 años es muy similar a la que registran los más jóvenes. Como era de esperar, la probabilidad de perder el empleo se reduce a medida que se incrementa la formación, en casi 1 punto porcentual en los niveles formativos elevados respecto a los más bajos. En el caso de la mujer, el impacto de esta variable es ligeramente superior al que se registra en el hombre. Estar casado también reduce la probabilidad de perder el empleo, aunque en mayor medida en los hombres que en las mujeres.

A nivel sectorial, el sector agrario es en el que resulta más probable perder el empleo, seguido del de la construcción. En el otro extremo se encuentra el sector industrial, donde la probabilidad de perder el empleo es en torno a 1 punto porcentual inferior respecto al sector de la construcción. Si bien este patrón se mantiene en el caso de los hombres, la muestra de mujeres resulta algo diferente, ya que, aunque el sector agrario sigue siendo el que registra una mayor probabilidad de perder el empleo (más de 5 puntos porcentuales superior a la del resto de sectores), en el sector de la construcción, industria y servicios, no se detectaron diferencias estadísticamente significativas.

Las variables asociadas al puesto de trabajo son las que más influyen en las diferencias detectadas en la probabilidad de perder el empleo entre inmigrantes y nativos. En concreto el tipo de contrato (indefinido versus temporal) y la antigüedad en la empresa, son las variables más relevantes, asociado este hecho a los menores costes de despido que tienen los contratos temporales, y los mayores costes, tanto económicos como de pérdida de capital humano, que tienen los contratos más antiguos. Así, la probabilidad de perder el empleo es 6,5 puntos porcentuales superior en un contrato temporal respecto a uno indefinido, y algo más de 3 puntos porcentuales en los contratos de menos de un año de antigüedad frente a los de más de tres años. Atendiendo al género, las diferencias en función del tipo de contrato se acentúa más entre las mujeres, donde la probabilidad de perder el empleo es 7,5 puntos porcentuales superior si la trabajadora tiene un contrato temporal.

El tipo de ocupación también resulta relevante para explicar la probabilidad de perder el empleo. En este caso, en las ocupaciones de mayor cualificación la probabilidad de perder el empleo es 1 punto porcentual inferior que en las de baja cualificación, siendo este efecto mayor entre las mujeres que entre los hombres. 


\section{CUADRO 2}

\section{ESTIMACIÓN LOGIT DE LA PROBABILIDAD DE PERDER EL EMPLEO. MUESTRA TOTAL}

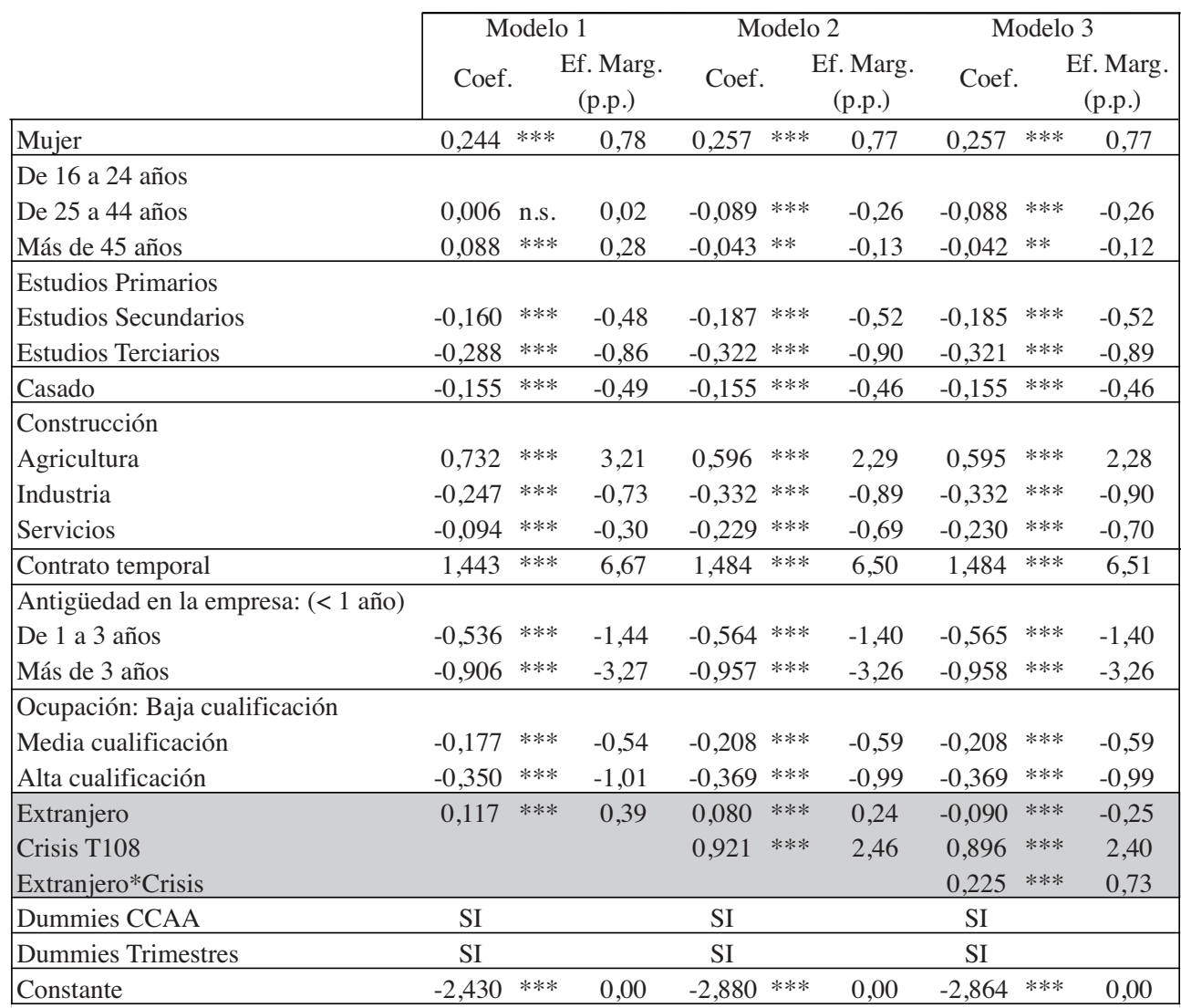

\begin{tabular}{|lccccc|}
\hline Número de observaciones & 847.173 & 847.173 & 847.173 \\
Test de la razón de verosimilitud & 59.096 & $* * *$ & 65.937 & $* * *$ & 65.974 \\
Pseudo R2 & 0,162 & & 0,181 & 0,181 \\
\hline
\end{tabular}

NOTA: El individuo de referencia es hombre, de 16 a 24 años de edad, con estudios primarios, casado, trabaja en el sector de la construcción, con un contrato temporal, una antigüedad en la empresa menor de 1 año y una ocupación de baja cualificación.

***,**,* denota coeficiente significativo al 1 por 100,5 por 100 y 10 por 100 respectivamente.

FUENTE: EPA (INE, 2005-2013). 


\section{CUADRO 3 \\ ESTIMACIÓN LOGIT DE LA PROBABILIDAD DE PERDER EL EMPLEO. MUESTRA HOMBRES}

\begin{tabular}{|c|c|c|c|c|c|c|c|c|c|}
\hline & \multicolumn{3}{|c|}{ Modelo 1} & \multicolumn{3}{|c|}{ Modelo 2} & \multicolumn{3}{|c|}{ Modelo 3} \\
\hline & \multicolumn{2}{|l|}{ Coef. } & \multirow[t]{2}{*}{$\begin{array}{c}\text { Ef. Marg. } \\
\text { (p.p.) }\end{array}$} & \multicolumn{2}{|c|}{ Coef. } & \multirow[t]{2}{*}{$\begin{array}{c}\text { Ef. Marg. } \\
\text { (p.p.) }\end{array}$} & \multicolumn{2}{|l|}{ Coef. } & $\begin{array}{c}\text { Ef. Marg. } \\
\text { (p.p.) }\end{array}$ \\
\hline De 16 a 24 años & & & & & & & & & \\
\hline De 25 a 44 años & 0,073 & $* * *$ & 0,21 & $-0,056$ & $* * *$ & $-0,14$ & $-0,054$ & $* * *$ & $-0,14$ \\
\hline Más de 45 años & 0,207 & $* * *$ & 0,61 & 0,043 & * & 0,11 & 0,045 & * & 0,12 \\
\hline \multicolumn{10}{|l|}{ Estudios Primarios } \\
\hline Estudios Secundarios & $-0,154$ & $* * *$ & $-0,42$ & $-0,186$ & $* * *$ & $-0,45$ & $-0,185$ & $* * *$ & $-0,45$ \\
\hline Estudios Terciarios & $-0,318$ & $* * *$ & $-0,86$ & $-0,346$ & $* * *$ & $-0,83$ & $-0,345$ & $* * *$ & $-0,83$ \\
\hline Casado & $-0,278$ & $* * *$ & $-0,82$ & $-0,285$ & **** & $-0,75$ & $-0,285$ & $* * *$ & $-0,75$ \\
\hline \multicolumn{10}{|l|}{ Construcción } \\
\hline Agricultura & 0,581 & $* * *$ & 2,15 & 0,393 & $* * *$ & 1,20 & 0,390 & $* * *$ & 1,19 \\
\hline Industria & $-0,305$ & $* * *$ & $-0,83$ & $-0,419$ & $* * *$ & $-0,99$ & $-0,419$ & $* * *$ & $-1,00$ \\
\hline Servicios & $-0,112$ & $* * *$ & $-0,32$ & $-0,284$ & $* * *$ & $-0,73$ & $-0,286$ & $* * *$ & $-0,73$ \\
\hline Contrato temporal & 1,401 & $* * *$ & 5,92 & 1,439 & **** & 5,53 & 1,439 & $* * *$ & 5,54 \\
\hline \multicolumn{10}{|l|}{ Antigüedad en la empresa: (< 1 año) } \\
\hline De 1 a 3 años & $-0,529$ & $* * *$ & $-1,28$ & $-0,553$ & $* * *$ & $-1,19$ & $-0,553$ & $* * *$ & $-1,19$ \\
\hline Más de 3 años & $-0,953$ & $* * *$ & $-3,25$ & $-1,006$ & $* * *$ & $-3,10$ & $-1,007$ & $* * *$ & $-3,11$ \\
\hline \multicolumn{10}{|l|}{ Ocupación: Baja cualificación } \\
\hline Media cualificación & $-0,051$ & $* *$ & $-0,14$ & $-0,092$ & $* * *$ & $-0,23$ & $-0,091$ & $* * *$ & $-0,23$ \\
\hline Alta cualificación & $-0,269$ & $* * *$ & $-0,72$ & $-0,300$ & $* * *$ & $-0,71$ & $-0,299$ & $* * *$ & $-0,71$ \\
\hline Extranjero & 0,256 & $* * *$ & 0,82 & 0,223 & $* * *$ & 0,63 & 0,050 & n.s. & 0,13 \\
\hline Crisis T108 & & & & 1,155 & $* * *$ & 2,72 & 1,129 & $* * *$ & 2,66 \\
\hline Extranjero*Crisis & & & & & & & 0,227 & $* * *$ & 0,64 \\
\hline Dummies CCAA & SI & & & SI & & & SI & & \\
\hline Dummies Trimestres & SI & & & SI & & & SI & & \\
\hline Constante & $-2,367$ & $* * *$ & 0,00 & $-2,923$ & $* * *$ & 0,00 & $-2,905$ & $* * *$ & 0,00 \\
\hline Número de observaciones & 483.233 & & & 483.23 & & & 483.233 & & \\
\hline Test de la razón de verosimilitud & 33.109 & & $* * *$ & 38.75 & & $* * *$ & 38.770 & & $* * *$ \\
\hline Pseudo R2 & 0,167 & & & 0,196 & & & 0,196 & & \\
\hline
\end{tabular}

NOTA: El individuo de referencia es de 16 a 24 años de edad, con estudios primarios, casado, trabaja en el sector de la construcción, con un contrato temporal, una antigüedad en la empresa menor de 1 año y una ocupación de baja cualificación.***, **, *denota coeficiente significativo al 1 por 100,5 por 100 y 10 por 100 respectivamente.

FUENTE: EPA (INE, 2005-2013). 


\section{CUADRO 4}

\section{ESTIMACIÓN LOGIT DE LA PROBABILIDAD DE PERDER EL EMPLEO. MUESTRA MUJERES}

\begin{tabular}{|c|c|c|c|c|c|c|c|c|c|}
\hline \multirow{3}{*}{ De 16 a 24 años } & \multicolumn{3}{|c|}{ Modelo 1} & \multicolumn{3}{|c|}{ Modelo 2} & \multicolumn{3}{|c|}{ Modelo 3} \\
\hline & \multicolumn{2}{|c|}{ Coef. } & $\begin{array}{c}\text { Ef. Marg. } \\
\text { (p.p.) }\end{array}$ & \multicolumn{2}{|c|}{ Coef. } & $\begin{array}{c}\text { Ef. Marg. } \\
\text { (p.p.) }\end{array}$ & \multicolumn{2}{|c|}{ Coef. } & $\begin{array}{c}\text { Ef. Marg. } \\
\text { (p.p.) }\end{array}$ \\
\hline & & & & & & & & & \\
\hline De 25 a 44 años & $-0,085$ & $* * *$ & $-0,30$ & $-0,144$ & $* * *$ & $-0,50$ & $-0,144$ & $* * *$ & $-0,50$ \\
\hline Más de 45 años & $-0,036$ & n.s. & $-0,13$ & $-0,129$ & $* * *$ & $-0,43$ & $-0,128$ & $* * *$ & $-0,43$ \\
\hline \multicolumn{10}{|l|}{ Estudios Primarios } \\
\hline Estudios Secundarios & $-0,160$ & $* * *$ & $-0,54$ & $-0,181$ & $* * *$ & $-0,59$ & $-0,179$ & $* * *$ & $-0,59$ \\
\hline Estudios Terciarios & $-0,247$ & $* * *$ & $-0,84$ & $-0,280$ & $* * *$ & $-0,92$ & $-0,278$ & $* * *$ & $-0,91$ \\
\hline Casado & $-0,044$ & $* * *$ & $-0,15$ & $-0,043$ & $* * *$ & $-0,15$ & $-0,042$ & $* *$ & $-0,14$ \\
\hline \multicolumn{10}{|l|}{ Construcción } \\
\hline Agricultura & 1,059 & $* * *$ & 6,11 & 1,015 & $* * *$ & 5,55 & 1,016 & $* * *$ & 5,56 \\
\hline Industria & $-0,082$ & n.s. & $-0,28$ & $-0,106$ & $*$ & $-0,35$ & $-0,105$ & $*$ & $-0,35$ \\
\hline Servicios & $-0,011$ & n.s. & $-0,04$ & $-0,076$ & n.s. & $-0,27$ & $-0,074$ & n.s. & $-0,26$ \\
\hline Contrato temporal & 1,469 & $* * *$ & 7,51 & 1,505 & $* * *$ & 7,53 & 1,505 & $* * *$ & 7,53 \\
\hline \multicolumn{10}{|l|}{ Antigüedad en la empresa: (< 1 año) } \\
\hline De 1 a 3 años & $-0,542$ & $* * *$ & $-1,64$ & $-0,569$ & $* * *$ & $-1,65$ & $-0,570$ & $* * *$ & $-1,66$ \\
\hline Más de 3 años & $-0,861$ & $* * *$ & $-3,35$ & $-0,908$ & $* * *$ & $-3,44$ & $-0,908$ & $* * *$ & $-3,44$ \\
\hline \multicolumn{10}{|l|}{ Ocupación: Baja cualificación } \\
\hline Media cualificación & $-0,267$ & $* * *$ & $-0,93$ & $-0,288$ & $* * *$ & $-0,97$ & $-0,288$ & $* * *$ & $-0,97$ \\
\hline Alta cualificación & $-0,462$ & $* * *$ & $-1,46$ & $-0,470$ & $* * *$ & $-1,44$ & $-0,470$ & $* * *$ & $-1,44$ \\
\hline Extranjero & $-0,034$ & n.s. & $-0,12$ & $-0,066$ & $* * *$ & $-0,22$ & $-0,252$ & $* * *$ & $-0,78$ \\
\hline Crisis T108 & & & & 0,661 & $* * *$ & 2,06 & 0,635 & $* * *$ & 1,98 \\
\hline Extranjero*Crisis & & & & & & & 0,251 & $* * *$ & 0,95 \\
\hline Dummies CCAA & SI & & & SI & & & SI & & \\
\hline Dummies Trimestres & SI & & & SI & & & SI & & \\
\hline Constante & $-2,267$ & $* * *$ & 0,00 & $-2,617$ & $* * *$ & 0,00 & $-2,603$ & $* * *$ & 0,00 \\
\hline Número de observaciones & 363.94 & & & 363.94 & & & 363.940 & & \\
\hline Test de la razón de verosimilitud & 26.28 & & $* * *$ & 27.95 & & $* * *$ & 27.973 & & $* * *$ \\
\hline Pseudo R2 & 0,158 & & & 0,168 & & & 0,168 & & \\
\hline
\end{tabular}

NOTA: El individuo de referencia es de 16 a 24 años de edad, con estudios primarios, casado, trabaja en el sector de la construcción, con un contrato temporal, una antigüedad en la empresa menor de 1 año y una ocupación de baja cualificación. $* * *, * * *$ denota coeficiente significativo al 1 por 100,5 por 100 y 10 por 100 respectivamente.

FUENTE: EPA (INE, 2005-2013). 
En resumen, las variables que más influyen en la probabilidad de perder el empleo son el tipo de contrato y la antigüedad en la empresa, seguidas, en menor medida, por el sector de actividad (siendo el agrario el que registra la mayor probabilidad de perder el empleo, seguido del sector de la construcción en el caso de los hombres), la formación y el tipo de ocupación. Atendiendo a las diferencias por género se observa que el impacto de estas variables sobre la probabilidad de perder el empleo es mayor en el caso de la mujer que del hombre.

En relación a las variables de interés, el modelo 1 incorpora como variable explicativa únicamente la nacionalidad del individuo. En este caso se observa que ser extranjero incrementa de forma significativa la probabilidad de perder el empleo, pero sólo en el caso de los hombres y en una cuantía de 0,8 puntos porcentuales. Cuando el efecto del ciclo económico sobre la probabilidad de perder el empleo es tenido en cuenta, modelo 2, los resultados son algo inferiores para el caso de los hombres, el impacto de ser extranjero es ahora de 0,6 puntos porcentuales, y pasan a ser significativos en el caso de las mujeres aunque a favor de la inmigrante, es decir, la probabilidad de perder el empleo es inferior en 0,2 puntos porcentuales en las extranjeras frente a las nativas.

Para ver si estas diferencias detectadas por nacionalidad tienen la misma intensidad en periodos de expansión y de crisis se incluye en la última especificación (modelo 3) la interacción entre ambas variables. El haber obtenido un resultado estadísticamente significativo, para el parámetro de la variable que recoge el término de la interacción, indica que las diferencias entre nativos y extranjeros no son las mismas según el momento del ciclo económico. El Gráfico 2 resume estos resultados a partir del efecto marginal obtenido para el periodo de expansión (parámetro de la variable extranjero) y para el periodo de crisis (suma del parámetro obtenido para la variable extranjero y para la interacción). 


\section{GRÁFICO 2}

\section{EFECTO MARGINAL SOBRE LA PROBABILIDAD DE PERDER EL EMPLEO POR SER EXTRANJERO}

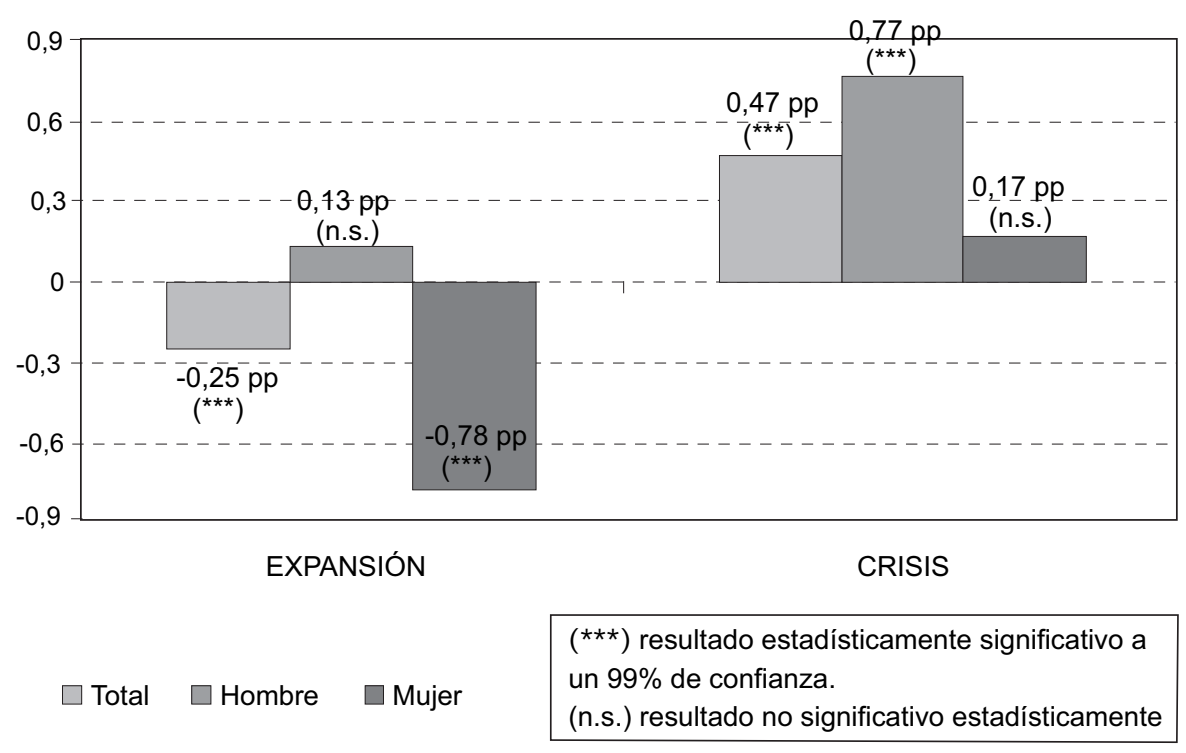

FUENTE: EPA (INE, 2005-2013).

Según los resultados de la muestra total, el efecto de la nacionalidad sobre la probabilidad de perder el empleo cambia de signo según el ciclo económico. Así, mientras que durante el periodo de expansión, ser inmigrante reduce la probabilidad de perder el empleo en 0,25 puntos porcentuales, durante el periodo de crisis se incrementa dicha probabilidad en 0,5 puntos porcentuales. Este hecho indicaría la existencia de una discriminación positiva a favor del inmigrante en épocas de expansión, que se duplica y se transforma en discriminación negativa durante la crisis.

Sin embargo este patrón no se mantiene cuando el análisis se diferencia según género, observándose la existencia de una discriminación positiva en las mujeres durante los ciclos expansivos, y negativa durante el periodo de crisis en el caso de los hombres.

En concreto, en el caso de la mujer se detecta una discriminación positiva a favor de la trabajadora inmigrante durante el periodo de expansión de 0,8 puntos porcentuales, no registrándose diferencias estadísticamente significativas debido a la nacionalidad durante el periodo de crisis. Dicha discriminación positiva podría estar justificada por el hecho de que el trabajo de la mujer inmigrante se concentra en el sector doméstico y de cuidado de ancianos, sectores en los que la nativa se ha visto desplazada por la inmigrante durante el periodo de expansión. En este sentido, la mayor disposición, por parte de las extranjeras, a aceptar trabajos peor remunerados y con peores condiciones laborales, condujo a la extranjerización del sector doméstico en los años previos a la crisis (Martínez, 2011). 
La situación es distinta para los trabajadores masculinos. Si bien durante el periodo de expansión económica, las diferencias detectadas en la probabilidad de perder el empleo entre inmigrantes y nativos se justifican por las distintas características laborales existentes entre ambos colectivos, al no obtenerse un parámetro estadísticamente significativo para la variable que mide la nacionalidad del individuo, esta situación no es la misma durante la crisis económica. En concreto, de la diferencia en la probabilidad de perder el empleo entre un hombre inmigrante y otro nativo en el periodo de crisis, cifrada en 7,4 puntos porcentuales (13,4 por 100 para el inmigrante versus 6 por 100 para el nativo), casi 1 punto porcentual (la suma del efecto marginal asociado a la variable extranjero y el asociado a la interacción) no se justifica por la existencia de diferentes características laborales en los colectivos, debiendo buscar las causas en otros factores no observables entre los que podría encontrarse la existencia de discriminación laboral negativa contra el trabajador inmigrante.

Si bien la cuantía de estos factores no observables no resulta elevada, sí es estadísticamente significativa y podría estar infravalorada, como ya se comentó, por el hecho de no poder diferenciar en la muestra la inmigración económica de la no económica. En el siguiente apartado se profundiza en el análisis de este tema.

\subsection{Descomposición de la diferencia de la probabilidad media de perder el empleo}

La descomposición de Oaxaca-Blinder, en su aproximación de Yun para formas funcionales no lineales, nos permite, tal y como se ha detallado en la descripción de la metodología, distinguir qué parte de la diferencia en la probabilidad de perder el empleo entre inmigrantes y nativos viene explicada por la existencia de diferencias en las características laborales entre ambos colectivos y cuál se debe a diferencias en la valoración que el mercado hace de dichas características, lo que podría considerarse como un comportamiento discriminatorio frente a uno de los colectivos. A su vez, esta metodología nos permite identificar qué factores son los que más influyen en dicho comportamiento. 
CUADRO 5

\begin{abstract}
DESCOMPOSICIÓN DE LA DIFERENCIA DE LA PROBABILIDAD MEDIA DE PERDER EL EMPLEO ENTRE INMIGRANTES Y NATIVOS. MUESTRA TOTAL
\end{abstract}

\begin{tabular}{|c|c|c|c|c|c|c|c|c|}
\hline & EXPANSIÓN & CRISIS & 2008 & 2009 & 2010 & 2011 & 2012 & 2013 \\
\hline \multicolumn{9}{|c|}{ Probabilidad de perder el empleo (puntos porcentuales): } \\
\hline Extranjero & $5,978 * * *$ & $11,414 * * *$ & $9,286 * * *$ & $12,298 * * *$ & $10,592 * * *$ & $11,253 * * *$ & $11,932 * * *$ & $11,022 * * *$ \\
\hline Nativo & $3,743 * * *$ & $6,141 * * *$ & $4,912 * * *$ & $5,992 * * *$ & $5,752 * * *$ & $5,917 * * *$ & $6,767 * * *$ & $6,319 * * *$ \\
\hline Diferencia & $2,235 * * *$ & $5,273 * * *$ & $4,374 * * *$ & $6,306 * * *$ & $4,840 * * *$ & $5,336 * * *$ & $5,164 * * *$ & $4,703 * * *$ \\
\hline \multicolumn{9}{|c|}{ Aportación a la diferencia en la probabilidad de perder el empleo de inmigrantes y nativos (puntos porcentuales): } \\
\hline \multirow[t]{2}{*}{ Parte explicada } & $2,298 * * *$ & $4,659 * * *$ & $3,781 * * *$ & $5,434 * * *$ & $4,363 * * *$ & $4,598 * * *$ & $4,659 * * *$ & $3,996 * * *$ \\
\hline & $(102,8 \%)$ & $(88,4 \%)$ & $(86,4 \%)$ & $(86,2 \%)$ & $(90,1 \%)$ & $(86,2 \%)$ & $(90,2 \%)$ & $(85 \%)$ \\
\hline \multirow[t]{2}{*}{ Género } & $0,091 * * *$ & $0,101 * * *$ & $0,090 * * *$ & $0,098 * * *$ & $0,131 * * *$ & $0,119 * * *$ & $0,079 * * *$ & $0,092 * * *$ \\
\hline & $(4,1 \%)$ & $(1,9$ & $(2,1 \%)$ & $(1,6 \%)$ & $(2,7 \%)$ & $(2,2 \%)$ & $(1,5 \%)$ & $(2 \%)$ \\
\hline \multirow[t]{2}{*}{ Edad } & $-0,036 * * *$ & $-0,040 * *$ & $-0,021$ n.s. & $-0,015$ n.s. & 0,008 n.s. & $-0,003$ n.s. & $-0,107 * *$ & $-0,046$ n.s. \\
\hline & $(-1,6 \%)$ & $(-0,8$ & $(-0,5 \%)$ & $(-0,2$ & $(0,2 \%)$ & $(-0,1 \%)$ & $(-2$, & $(-1 \%)$ \\
\hline \multirow[t]{2}{*}{ Formación } & $-0,040 * * *$ & $0,111 * * *$ & $-0,002$ n.s. & $0,109 * *$ & $0,061^{*}$ & $0,104 * *$ & $0,096 * *$ & $0,219 * * *$ \\
\hline & $(-1,8$ & $(2,1 \%)$ & $(0 \%)$ & $(1,7$ & $(1,3 \%)$ & $(2$ & $(1,9$ & $(4,7 \%)$ \\
\hline \multirow[t]{2}{*}{ Estado civil } & $0,001 \mathrm{n} . \mathrm{s}$. & $0,033 * * *$ & 0,006 n.s. & $0,048 * * *$ & $0,026 * * *$ & $0,030 * * *$ & $0,032 * * *$ & $0,023 * * *$ \\
\hline & & $(0,6$ & $(0,1 \%)$ & $(0,8$ & $(0,5 \%)$ & $(0,6$ & $(0$, & $(0,5 \%)$ \\
\hline \multirow[t]{2}{*}{ Sector actividad } & $0,098 * * *$ & $0,410 * * *$ & $0,282 * * *$ & $0,346 * * *$ & $0,362 * * *$ & $0,404 * * *$ & $0,443 * * *$ & $0,518 * * *$ \\
\hline & $(4,4 \%)$ & $(7,8$ & $(6,4 \%)$ & $(5,5$ & $(7,5 \%)$ & $(7,6$ & $(8,6$ & $(11 \%)$ \\
\hline \multirow[t]{2}{*}{ Tipo de contrato } & $1,215 * * *$ & $1,981 * * *$ & $1,558 * * *$ & $2,196 * * *$ & $1,815 * * *$ & $2,012 * * *$ & $2,009 * * *$ & $1,667 * * *$ \\
\hline & $(54,4 \%)$ & $(37,6 \%)$ & $(35,6 \%)$ & $(34,8 \%)$ & $(37,5 \%)$ & $(37,7 \%)$ & $(38,9 \%)$ & $(35,5 \%)$ \\
\hline \multirow[t]{2}{*}{ Atiguedad en empresa } & a $\quad 0,884 * * *$ & $1,476 * * *$ & $1,534 * * *$ & $1,890 * * *$ & $1,472 * * *$ & $1,349 * * *$ & $1,454 * * *$ & $1,168 * * *$ \\
\hline & $(39,6 \%)$ & $(28 \%)$ & $(35,1 \%)$ & $(30 \%)$ & $(30,4 \%)$ & $(25,3 \%)$ & $(28,1 \%)$ & $(24,8 \%)$ \\
\hline \multirow[t]{2}{*}{ Ocupación } & $0,188 * * *$ & $0,537 * * *$ & $0,414 * * *$ & $0,609 * * *$ & $0,432 * * *$ & $0,577 * * *$ & 0,651 *** & $0,371 * * *$ \\
\hline & $(8,4 \%)$ & $(10,2 \%)$ & $(9,5 \%)$ & $(9,7 \%)$ & $(8,9 \%)$ & $(10,8 \%)$ & $(12$, & $(7,9 \%)$ \\
\hline \multirow[t]{2}{*}{ CCAA } & $-0,107 * * *$ & $0,046^{*}$ & $-0,102 * *$ & $* * *$ & 0,057 n.s. & 0,010 n.s. & $-0,004$ n.s. & $-0,026$ n.s. \\
\hline & $(-4,8 \%)$ & $(0,9 \%)$ & $(-2,3$ & $(2,4$ & $(1,2 \%)$ & $(0,2 \%)$ & $(-0$, & $(-0,5 \%)$ \\
\hline \multirow[t]{2}{*}{ Estacionalidad } & $0,005 * * *$ & n.s. & 0,021 n.s. & 0,004 n.s. & $-0,001$ n.s. & 4 n.s. & n.s. & 0,008 n.s. \\
\hline & $(0,2 \%)$ & $(0,1$ & $(0$, & $(0,1 \%)$ & $(0 \%)$ & $(-0,1$ & $(0$, & $(0,2 \%)$ \\
\hline \multirow[t]{2}{*}{ Parte no explicada } & $-0,063$ n.s. & $0,614 * * *$ & $0,594 * * *$ & $0,871 * * *$ & $0,477 * *$ & $0,738 * * *$ & $0,505 *$ & $0,707 * * *$ \\
\hline & $(-2,8 \%)$ & $(11,6 \%)$ & $(13,6 \%)$ & $(13,8 \%)$ & $(9,9 \%)$ & $(13,8 \%)$ & $(9,8 \%)$ & $(15 \%)$ \\
\hline \multirow[t]{2}{*}{ Género } & $-0,005$ n.s. & $-0,004 * * *$ & $0,029 * * *$ & $0,014 * *$ & $-0,002$ n.s. & $-0,007 *$ & $-0,007$ n.s. & $-0,020 * *$ \\
\hline & $(-0,2 \%)$ & $(-0,1 \%)$ & $(0,7 \%)$ & $(0,2 \%)$ & $(0 \%)$ & $(-0,1 \%)$ & $(-0$, & $(-0,4 \%)$ \\
\hline \multirow[t]{2}{*}{ Edad } & 0,005 n.s. & $-0,111 * * *$ & 0,003 n.s. & $-0,081$ n.s. & 0,030 n.s. & $-0,123 *$ & $-0,251 * *$ & $-0,245 * *$ \\
\hline & $(0,2 \%)$ & $(-2$, & $(0,1 \%)$ & $(-1,3$ & $(0,6 \%)$ & $(-2$, & $(-4$ & \\
\hline \multirow[t]{2}{*}{ Formación } & $-0,001 \mathrm{n} . \mathrm{s}$ & $-0,035 * * *$ & $-0,027$ n.s. & $-0,065 * *$ & $-0,022$ n.s. & $-0,042 *$ & $-0,056^{*}$ & $-0,022 \mathrm{n} . \mathrm{s}$. \\
\hline & $(-0,1 \%)$ & $(-0,7$ & $(-0,6 \%)$ & & $(-0,5 \%)$ & $(-0,8 \%)$ & $(-1,1 \%)$ & $(-0,5 \%)$ \\
\hline \multirow[t]{2}{*}{ Estado civil } & $-0,002$ n.s. & $0,022 * * *$ & 0,012 n.s. & $0,030 * * *$ & $0,015 *$ & 0,007 n.s. & $0,037 * *$ & $0,032 *$ \\
\hline & $(-0,1 \%)$ & $(0,4 \%)$ & $(0,3 \%)$ & $(0,5 \%)$ & $(0,3 \%)$ & $(0,1 \%)$ & $(0,7 \%)$ & $(0,7 \%)$ \\
\hline \multirow[t]{2}{*}{ Sector actividad } & $-0,027$ n.s. & 0,041 n.s. & $0,183 * *$ & $0,250 * *$ & $-0,062$ n.s. & 0,015 n.s. & $0,036 \mathrm{n} . \mathrm{s}$. & 0,004 n.s. \\
\hline & $(-1,2 \%)$ & $(0,8 \%)$ & & & $(-1,3 \%)$ & & & $(0,1 \%)$ \\
\hline Tipo de contrato & 0,007 n.s. & $0,111 * * *$ & $0,025 * *$ & $0,065 * * *$ & $0,109 * * *$ & $0,143 * * *$ & $0,111 * *$ & $0,189 * * *$ \\
\hline & $(0,3 \%)$ & $(2,1 \%)$ & $(0,6 \%)$ & & & & & $(4 \%)$ \\
\hline Atiguedad en empresa & a 0,009 n.s. & $0,031 * * *$ & $-0,043 * * *$ & $-0,001$ n.s. & 0,013 n.s. & 0,016 n.s. & 0,023 n.s. & 0,021 n.s. \\
\hline & $(0,4 \%)$ & $(0,6 \%)$ & & & & & & $(0,5 \%)$ \\
\hline Ocupación & 0,012 n.s. & $-0,037$ n.s. & $-0,153$ n.s. & $-0,073$ n.s. & $-0,006$ n.s. & $-0,003$ n.s. & $-0,039$ n.s. & $-0,084$ n.s. \\
\hline & $(0,5 \%)$ & $(-0,7 \%)$ & $(-3,5 \%)$ & $(-1,2 \%)$ & $(-0,1 \%)$ & $(-0,1 \%)$ & $(-0,8 \%)$ & $(-1,8 \%)$ \\
\hline CCAA & 0,008 n.s. & 0,008 n.s. & 0,113 n.s. & 0,106 n.s. & $-0,058$ n.s. & 0,092 n.s. & $-0,042$ n.s. & $-0,037$ n.s. \\
\hline & & & & & $(-1,2 \%)$ & & & $(-0,8 \%)$ \\
\hline Estacionalidad & 0,001 n.s. & $0,000 \mathrm{n} . \mathrm{s}$. & $0,001 \mathrm{n} . \mathrm{s}$. & $0,007 *$ & $-0,002$ n.s. & $0,001 \mathrm{n} . \mathrm{s}$. & 0,000 n.s. & 0,000 n.s. \\
\hline & $(0 \%)$ & & & $(0,1 \%)$ & & & & \\
\hline Constante & $-0,069$ n.s. & $0,587 * * *$ & $0,451 * *$ & $0,620 * *$ & $0,461 *$ & $0,639 * *$ & $0,693 *$ & $0,868 * *$ \\
\hline & $(-3,1 \%)$ & $(11,1 \%)$ & $(10,3 \%)$ & $(9,8 \%)$ & $(9,5 \%)$ & $(12 \%)$ & $(13,4 \%)$ & $(18,4 \%)$ \\
\hline
\end{tabular}

NOTA: Los valores entre paréntesis indican el peso de la contribución a la diferencia en la probabilidad de perder el empleo entre inmigrantes y nativos. $* * *, * *, *$ denota valores significativos al 1 por 100,5 por 100 y 10 por 100 respectivamente.

FUENTE: EPA (INE, 2005-2013). 


\section{CUADRO 6}

\section{DESCOMPOSICIÓN DE LA DIFERENCIA DE LA PROBABILIDAD MEDIA DE PERDER EL EMPLEO ENTRE INMIGRANTES Y NATIVOS. MUESTRA DE HOMBRES.}

\begin{tabular}{|c|c|c|c|c|c|c|c|c|}
\hline & EXPANSIÓN & CRISIS & 2008 & 2009 & 2010 & 2011 & 2012 & 2013 \\
\hline \multicolumn{9}{|c|}{ Probabilidad de perder el empleo (puntos porcentuales): } \\
\hline Extranjero & $5,734 * * *$ & $13,361 * * *$ & $10,667 * * *$ & $14,516 * * *$ & $12,024 * * *$ & $13,247 * * *$ & $13,989 * * *$ & $13,046 * * *$ \\
\hline Nativo & $3,020 * * *$ & $6,011 * * *$ & $4,611 * * *$ & $5,851 * * *$ & $5,560 * * *$ & $5,828 * * *$ & $6,754 * * *$ & $6,136 * * *$ \\
\hline Diferencia & $2,715 * * *$ & $7,350 * * *$ & $6,056 * * *$ & $8,664 * * *$ & $6,464 * * *$ & $7,419 * * *$ & $7,235 * * *$ & $6,911 * * *$ \\
\hline \multicolumn{9}{|c|}{ Aportación a la diferencia en la probabilidad de perder el empleo de inmigrantes y nativos (puntos porcentuales): } \\
\hline Parte explicada & $\begin{array}{l}2,189 * * * \\
(80,6 \%)\end{array}$ & $\begin{array}{l}6,041 * * * \\
(82,2 \%)\end{array}$ & $\begin{array}{l}4,862 * * * \\
(80,3 \%)\end{array}$ & $\begin{array}{l}7,107 \text { *** } \\
(82 \%)\end{array}$ & $\begin{array}{l}5,538 * * * \\
(85,7 \%)\end{array}$ & $\begin{array}{l}5,948 * * * \\
(80,2 \%)\end{array}$ & $\begin{array}{l}6,106 * * * \\
(84,4 \%)\end{array}$ & $\begin{array}{l}5,036 \text { *** } \\
(72,9 \%)\end{array}$ \\
\hline Edad & $\begin{array}{l}-0,080 \text { *** } \\
(-3 \%)\end{array}$ & $\begin{array}{l}-0,141 * * * \\
(-1,9 \%)\end{array}$ & $\begin{array}{l}-0,053 \text { n.s. } \\
(-0,9 \%)\end{array}$ & $\begin{array}{l}-0,073 \text { n.s. } \\
(-0,8 \%)\end{array}$ & $\begin{array}{l}-0,050 \text { n.s. } \\
(-0,8 \%)\end{array}$ & $\begin{array}{l}-0,123 \text { n.s. } \\
(-1,7 \%)\end{array}$ & $\begin{array}{l}-0,263 \text { *** } \\
(-3,6 \%)\end{array}$ & $\begin{array}{l}-0,147 \text { ** } \\
(-2,1 \%)\end{array}$ \\
\hline Formación & $\begin{array}{l}-0,060 * * * \\
(-2,2 \%)\end{array}$ & $\begin{array}{l}0,125 * * * \\
(1,7 \%)\end{array}$ & $\begin{array}{l}-0,053 \text { n.s. } \\
(-0,9 \%)\end{array}$ & $\begin{array}{l}0,203 * * * \\
(2,3 \%)\end{array}$ & $\begin{array}{l}0,062 \text { n.s. } \\
(1 \%)\end{array}$ & $\begin{array}{c}0,074 \text { n.s. } \\
(1 \%)\end{array}$ & $\begin{array}{l}0,066 \text { n.s. } \\
(0,9 \%)\end{array}$ & $\begin{array}{l}0,252 \text { *** } \\
(3,6 \%)\end{array}$ \\
\hline Estado civil & $\begin{array}{l}-0,004 \text { n.s. } \\
(-0,1 \%)\end{array}$ & $\begin{array}{l}-0,018 * * \\
(-0,2 \%)\end{array}$ & $\begin{array}{l}-0,009 \text { n.s. } \\
(-0,1 \%)\end{array}$ & $\begin{array}{l}0,054 \text { ** } \\
(0,6 \%)\end{array}$ & $\begin{array}{l}0,008 \text { n.s. } \\
(0,1 \%)\end{array}$ & $\begin{array}{l}-0,039 * * \\
(-0,5 \%)\end{array}$ & $\begin{array}{l}-0,059 * * * \\
(-0,8 \%)\end{array}$ & $\begin{array}{c}-0,068 \text { *** } \\
(-1 \%)\end{array}$ \\
\hline Sector actividad & $\begin{array}{l}0,100 * * * \\
(3,7 \%)\end{array}$ & $\begin{array}{l}0,895 * * * \\
(12,2 \%)\end{array}$ & $\begin{array}{l}0,579 * * * \\
(9,6 \%)\end{array}$ & $\begin{array}{l}0,927 \text { *** } \\
(10,7 \%)\end{array}$ & $\begin{array}{c}0,816 * * * \\
(12,6 \%)\end{array}$ & $\begin{array}{l}0,813 * * * \\
(11 \%)\end{array}$ & $\begin{array}{l}0,935 \text { *** } \\
(12,9 \%)\end{array}$ & $\begin{array}{l}1,049 \text { *** } \\
(15,2 \%)\end{array}$ \\
\hline Tipo de contrato & $\begin{array}{l}1,284 \text { **** } \\
(47,3 \%)\end{array}$ & $\begin{array}{l}2,824 * * * \\
(38,4 \%)\end{array}$ & $\begin{array}{l}2,163 * * * \\
(35,7 \%)\end{array}$ & $\begin{array}{l}2,807 \text { *** } \\
(32,4 \%)\end{array}$ & $\begin{array}{c}2,600 * * * \\
(40,2 \%)\end{array}$ & $\begin{array}{l}3,076 * * * \\
(41,5 \%)\end{array}$ & $\begin{array}{l}2,972 \text { *** } \\
(41,1 \%)\end{array}$ & $\begin{array}{l}2,390 \text { *** } \\
(34,6 \%)\end{array}$ \\
\hline Atiguedad en empresa & $\begin{array}{l}0,960 * * * \\
(35,4 \%)\end{array}$ & $\begin{array}{l}1,881 * * * \\
(25,6 \%)\end{array}$ & $\begin{array}{l}1,846 * * * \\
(30,5 \%)\end{array}$ & $\begin{array}{l}2,576 * * * \\
(29,7 \%)\end{array}$ & $\begin{array}{l}1,757 * * * \\
(27,2 \%)\end{array}$ & $\begin{array}{l}1,722 * * * \\
(23,2 \%)\end{array}$ & $\begin{array}{l}1,752 * * * \\
(24,2 \%)\end{array}$ & $\begin{array}{l}1,370 \text { *** } \\
(19,8 \%)\end{array}$ \\
\hline Ocupación & $\begin{array}{l}0,080 \text { *** } \\
(3 \%)\end{array}$ & $\begin{array}{l}0,444 * * * \\
(6 \%)\end{array}$ & $\begin{array}{l}0,367 * * * \\
(6,1 \%)\end{array}$ & $\begin{array}{l}0,420 \text { *** } \\
(4,9 \%)\end{array}$ & $\begin{array}{l}0,321 * * * \\
(5 \%)\end{array}$ & $\begin{array}{l}0,478 * * * \\
(6,4 \%)\end{array}$ & $\begin{array}{l}0,669 * * * \\
(9,2 \%)\end{array}$ & $\begin{array}{l}0,357 \text { *** } \\
(5,2 \%)\end{array}$ \\
\hline CCAA & $\begin{array}{l}-0,095 * * * \\
(-3,5 \%)\end{array}$ & $\begin{array}{l}0,028 \text { n.s. } \\
(0,4 \%)\end{array}$ & $\begin{array}{l}0,024 \text { n.s. } \\
(0,4 \%)\end{array}$ & $\begin{array}{l}0,185 * \\
(2,1 \%)\end{array}$ & $\begin{array}{l}0,020 \text { n.s. } \\
(0,3 \%)\end{array}$ & $\begin{array}{l}-0,040 \text { n.s. } \\
(-0,5 \%)\end{array}$ & $\begin{array}{l}0,032 \text { n.s. } \\
(0,4 \%)\end{array}$ & $\begin{array}{l}-0,174 \text { n.s. } \\
(-2,5 \%)\end{array}$ \\
\hline Estacionalidad & $\begin{array}{l}0,003 \text { n.s. } \\
(0,1 \%)\end{array}$ & $\begin{array}{l}0,003 \text { n.s. } \\
(0 \%)\end{array}$ & $\begin{array}{l}-0,002 \text { n.s. } \\
(0 \%)\end{array}$ & $\begin{array}{l}0,008 \text { n.s. } \\
(0,1 \%)\end{array}$ & $\begin{array}{l}0,005 \text { n.s. } \\
(0,1 \%)\end{array}$ & $\begin{array}{l}-0,012 \text { n.s. } \\
(-0,2 \%)\end{array}$ & $\begin{array}{l}0,003 \text { n.s. } \\
(0 \%)\end{array}$ & $\begin{array}{l}0,008 \text { n.s. } \\
(0,1 \%)\end{array}$ \\
\hline Parte no explicada & $\begin{array}{l}0,526 * * * \\
(19,4 \%)\end{array}$ & $\begin{array}{l}1,309 * * * \\
(17,8 \%)\end{array}$ & $\begin{array}{l}1,193 * * * \\
(19,7 \%)\end{array}$ & $\begin{array}{l}1,557 * * * \\
(18 \%)\end{array}$ & $\begin{array}{l}0,926 * * * \\
(14,3 \%)\end{array}$ & $\begin{array}{l}1,471 * * * \\
(19,8 \%)\end{array}$ & $\begin{array}{l}1,129 * * * \\
(15,6 \%)\end{array}$ & $\begin{array}{l}1,875 \text { *** } \\
(27,1 \%)\end{array}$ \\
\hline Edad & $\begin{array}{l}-0,021 \text { n.s. } \\
(-0,8 \%)\end{array}$ & $\begin{array}{l}-0,182 * * * \\
(-2,5 \%)\end{array}$ & $\begin{array}{l}-0,050 \text { n.s. } \\
(-0,8 \%)\end{array}$ & $\begin{array}{l}-0,222 \text { n.s. } \\
(-2,6 \%)\end{array}$ & $\begin{array}{l}-0,058 \text { n.s. } \\
(-0,9 \%)\end{array}$ & $\begin{array}{l}-0,183 \text { n.s. } \\
(-2,5 \%)\end{array}$ & $\begin{array}{l}-0,318 \text { n.s. } \\
(-4,4 \%)\end{array}$ & $\begin{array}{l}-0,195 \text { n.s. } \\
(-2,8 \%)\end{array}$ \\
\hline Formación & $\begin{array}{l}0,004 \text { n.s. } \\
(0,2 \%)\end{array}$ & $\begin{array}{l}-0,071 * * * \\
(-1 \%)\end{array}$ & $\begin{array}{l}-0,043 \text { n.s. } \\
(-0,7 \%)\end{array}$ & $\begin{array}{l}-0,096 \text { n.s. } \\
(-1,1 \%)\end{array}$ & $\begin{array}{l}-0,084 * \\
(-1,3 \%)\end{array}$ & $\begin{array}{l}-0,006 \mathrm{n} . \mathrm{s} . \\
(-0,1 \%)\end{array}$ & $\begin{array}{l}-0,141 * * \\
(-2 \%)\end{array}$ & $\begin{array}{l}-0,076 \text { n.s. } \\
(-1,1 \%)\end{array}$ \\
\hline Estado civil & $\begin{array}{c}0,027 * * \\
(1 \%)\end{array}$ & $\begin{array}{l}0,059 * * * \\
(0,8 \%)\end{array}$ & $\begin{array}{l}0,048 * \\
(0,8 \%)\end{array}$ & $\begin{array}{l}0,086 \text { *** } \\
(1 \%)\end{array}$ & $\begin{array}{l}0,026 \text { n.s. } \\
(0,4 \%)\end{array}$ & $\begin{array}{l}-0,031 \text { n.s. } \\
(-0,4 \%)\end{array}$ & $\begin{array}{l}0,079 \text { n.s. } \\
(1,1 \%)\end{array}$ & $\begin{array}{c}0,136 * \\
(2 \%)\end{array}$ \\
\hline Sector actividad & $\begin{array}{l}0,106 * * * \\
(3,9 \%)\end{array}$ & $\begin{array}{l}0,018 \text { n.s. } \\
(0,2 \%)\end{array}$ & $\begin{array}{l}0,154 * \\
(2,5 \%)\end{array}$ & $\begin{array}{l}0,209 * * \\
(2,4 \%)\end{array}$ & $\begin{array}{l}-0,092 \text { n.s. } \\
(-1,4 \%)\end{array}$ & $\begin{array}{l}-0,014 \text { n.s. } \\
(-0,2 \%)\end{array}$ & $\begin{array}{l}-0,034 \text { n.s. } \\
(-0,5 \%)\end{array}$ & $\begin{array}{l}-0,045 \text { n.s. } \\
(-0,7 \%)\end{array}$ \\
\hline Tipo de contrato & $\begin{array}{l}-0,054 * * * \\
(-2 \%)\end{array}$ & $\begin{array}{l}0,121 * * * \\
(1,6 \%)\end{array}$ & $\begin{array}{l}-0,006 \text { n.s. } \\
(-0,1 \%)\end{array}$ & $\begin{array}{l}0,057 * \\
(0,7 \%)\end{array}$ & $\begin{array}{l}0,113 * * * \\
(1,8 \%)\end{array}$ & $\begin{array}{l}0,126 * * * \\
(1,7 \%)\end{array}$ & $\begin{array}{l}0,187 * * * \\
(2,6 \%)\end{array}$ & $\begin{array}{l}0,214 * * \\
(3,1 \%)\end{array}$ \\
\hline Atiguedad en empresa & $\begin{array}{l}-0,041 * * * \\
(-1,5 \%)\end{array}$ & $\begin{array}{l}0,063 * * \\
(0,9 \%)\end{array}$ & $\begin{array}{l}-0,024 \text { n.s. } \\
(-0,4 \%)\end{array}$ & $\begin{array}{l}0,027 \text { n.s. } \\
(0,3 \%)\end{array}$ & $\begin{array}{l}0,043 \text { n.s. } \\
(0,7 \%)\end{array}$ & $\begin{array}{l}0,035 \text { n.s. } \\
(0,5 \%)\end{array}$ & $\begin{array}{l}-0,025 \text { n.s. } \\
(-0,3 \%)\end{array}$ & $\begin{array}{l}0,171 \text { n.s. } \\
(2,5 \%)\end{array}$ \\
\hline Ocupación & $\begin{array}{l}0,058 \text { n.s. } \\
(2,1 \%)\end{array}$ & $\begin{array}{l}0,045 \text { n.s. } \\
(0,6 \%)\end{array}$ & $\begin{array}{l}-0,237 \text { n.s. } \\
(-3,9 \%)\end{array}$ & $\begin{array}{l}0,299 \text { n.s. } \\
(3,4 \%)\end{array}$ & $\begin{array}{l}0,180 \text { n.s. } \\
(2,8 \%)\end{array}$ & $\begin{array}{l}-0,138 \text { n.s. } \\
(-1,9 \%)\end{array}$ & $\begin{array}{l}0,060 \text { n.s. } \\
(0,8 \%)\end{array}$ & $\begin{array}{l}-0,173 \text { n.s. } \\
(-2,5 \%)\end{array}$ \\
\hline CCAA & $\begin{array}{l}-0,044 \text { n.s. } \\
(-1,6 \%)\end{array}$ & $\begin{array}{c}0,074 \text { n.s. } \\
(1 \%)\end{array}$ & $\begin{array}{l}0,203 \text { n.s. } \\
(3,4 \%)\end{array}$ & $\begin{array}{l}0,078 \text { n.s. } \\
(0,9 \%)\end{array}$ & $\begin{array}{l}-0,004 \text { n.s. } \\
(-0,1 \%)\end{array}$ & $\begin{array}{l}0,081 \text { n.s. } \\
(1,1 \%)\end{array}$ & $\begin{array}{l}0,150 \text { n.s. } \\
(2,1 \%)\end{array}$ & $\begin{array}{l}0,187 \text { n.s. } \\
(2,7 \%)\end{array}$ \\
\hline Estacionalidad & $\begin{array}{l}0,005 \text { n.s. } \\
(0,2 \%)\end{array}$ & $\begin{array}{c}0,002 \text { n.s. } \\
(0 \%)\end{array}$ & $\begin{array}{l}0,008 \text { n.s. } \\
(0,1 \%)\end{array}$ & $\begin{array}{l}0,008 \text { n.s. } \\
(0,1 \%)\end{array}$ & $\begin{array}{c}-0,002 \text { n.s. } \\
(0 \%)\end{array}$ & $\begin{array}{l}0,002 \text { n.s. } \\
(0 \%)\end{array}$ & $\begin{array}{l}0,004 \text { n.s. } \\
(0,1 \%)\end{array}$ & $\begin{array}{l}0,006 \text { n.s. } \\
(0,1 \%)\end{array}$ \\
\hline Constante & $\begin{array}{l}0,485 * * * \\
(17,9 \%)\end{array}$ & $\begin{array}{c}1,181 * * * \\
(16,1 \%)\end{array}$ & $\begin{array}{l}1,141 * * * \\
(18,8 \%)\end{array}$ & $\begin{array}{l}1,113 \text { **** } \\
(12,8 \%)\end{array}$ & $\begin{array}{l}0,803 \\
(12,4 \%)\end{array}$ & $\begin{array}{l}1,599 * * * \\
(21,6 \%)\end{array}$ & $\begin{array}{l}1,167 * * \\
(16,1 \%)\end{array}$ & $\begin{array}{l}1,649 \text { *** } \\
(23,9 \%)\end{array}$ \\
\hline
\end{tabular}

NOTA: Los valores entre paréntesis indican el peso de la contribución a la diferencia en la probabilidad de perder el empleo entre inmigrantes y nativos. $* * *, * *, *$ denota valores significativos al 1 por 100,5 por 100 y 10 por 100 respectivamente.

FUENTE: EPA (INE, 2005-2013). 
CUADRO 7

\section{DESCOMPOSICIÓN DE LA DIFERENCIA DE LA PROBABILIDAD MEDIA DE PERDER EL EMPLEO ENTRE INMIGRANTES Y NATIVOS. MUESTRA DE MUJERES}

\begin{tabular}{|c|c|c|c|c|c|c|c|c|}
\hline & EXPANSIÓN & CRISIS & 2008 & 2009 & 2010 & 2011 & 2012 & 2013 \\
\hline \multicolumn{9}{|c|}{ Probabilidad de perder el empleo (puntos porcentuales): } \\
\hline Extranjero & $6,272 * * *$ & $9,600 * * *$ & $7,717 * * *$ & $10,045 * * *$ & $9,310 * * *$ & $9,447 * * *$ & $10,102 * * *$ & $9,248 * * *$ \\
\hline Nativo & $4,864 * * *$ & $6,303 * * *$ & $5,346 * * *$ & $6,180 * * *$ & $6,003 * * *$ & $6,029 * * *$ & $6,783 * * *$ & $6,533 * * *$ \\
\hline Diferencia & $1,408 * * *$ & $3,296 * * *$ & $2,371 * * *$ & $3,866 * * *$ & $3,307 * * *$ & $3,418 * * *$ & $3,318 * * *$ & $2,715 * * *$ \\
\hline \multicolumn{9}{|c|}{ Aportación a la diferencia en la probabilidad de perder el empleo de inmigrantes y nativos (puntos porcentuales): } \\
\hline Parte explicada & $\begin{array}{l}2,191 * * * \\
(155,6 \%)\end{array}$ & $\begin{array}{c}3,405 * * * \\
(103,3 \%)\end{array}$ & $\begin{array}{l}2,515 * * * \\
(106,1 \%)\end{array}$ & $\begin{array}{l}3,722 * * * \\
(96,3 \%)\end{array}$ & $\begin{array}{l}3,263 * * * \\
(98,7 \%)\end{array}$ & $\begin{array}{l}3,402 * * * \\
(99,5 \%)\end{array}$ & $\begin{array}{c}3,507 \text { *** } \\
(105,7 \%)\end{array}$ & $\begin{array}{c}3,078 \text { *** } \\
(113,4 \%)\end{array}$ \\
\hline Edad & $\begin{array}{l}-0,020 * * \\
(-1,4 \%)\end{array}$ & $\begin{array}{l}0,006 \text { n.s. } \\
(0,2 \%)\end{array}$ & $\begin{array}{l}-0,028 \text { n.s. } \\
(-1,2 \%)\end{array}$ & $\begin{array}{l}-0,007 \text { n.s. } \\
(-0,2 \%)\end{array}$ & $\begin{array}{l}0,016 \text { n.s. } \\
(0,5 \%)\end{array}$ & $\begin{array}{l}0,048 \text { n.s. } \\
(1,4 \%)\end{array}$ & $\begin{array}{l}-0,013 \text { n.s. } \\
(-0,4 \%)\end{array}$ & $\begin{array}{l}0,005 \text { n.s. } \\
(0,2 \%)\end{array}$ \\
\hline Formación & $\begin{array}{l}-0,010 * * * \\
(-0,7 \%)\end{array}$ & $\begin{array}{l}0,108 * * * \\
(3,3 \%)\end{array}$ & $\begin{array}{l}0,065 \text { n.s. } \\
(2,7 \%)\end{array}$ & $\begin{array}{l}0,036 \mathrm{n} . \mathrm{s} . \\
(0,9 \%)\end{array}$ & $\begin{array}{l}0,072 \text { n.s. } \\
(2,2 \%)\end{array}$ & $\begin{array}{c}0,137 * * \\
(4 \%)\end{array}$ & $\begin{array}{l}0,144 * * \\
(4,3 \%)\end{array}$ & $\begin{array}{l}0,202 * * * \\
(7,5 \%)\end{array}$ \\
\hline Estado civil & $\begin{array}{c}0,000 \text { *** } \\
(0 \%)\end{array}$ & $\begin{array}{l}0,022 * * * \\
(0,7 \%)\end{array}$ & $\begin{array}{l}0,001 \text { n.s. } \\
(0 \%)\end{array}$ & $\begin{array}{l}0,024 \text { ** } \\
(0,6 \%)\end{array}$ & $\begin{array}{l}-0,004 \text { n.s. } \\
(-0,1 \%)\end{array}$ & $\begin{array}{l}0,015 \text { n.s. } \\
(0,4 \%)\end{array}$ & $\begin{array}{l}0,030 * \\
(0,9 \%)\end{array}$ & $\begin{array}{l}0,035 * \\
(1,3 \%)\end{array}$ \\
\hline Sector actividad & $\begin{array}{l}0,092 * * \\
(6,5 \%)\end{array}$ & $\begin{array}{l}0,087 * * * \\
(2,6 \%)\end{array}$ & $\begin{array}{l}0,055 * * \\
(2,3 \%)\end{array}$ & $\begin{array}{l}-0,027 \text { n.s. } \\
(-0,7 \%)\end{array}$ & $\begin{array}{l}0,060 \text { n.s. } \\
(1,8 \%)\end{array}$ & $\begin{array}{l}0,126 * * * \\
(3,7 \%)\end{array}$ & $\begin{array}{l}0,124 * * * \\
(3,7 \%)\end{array}$ & $\begin{array}{l}0,130 \text { *** } \\
(4,8 \%)\end{array}$ \\
\hline Tipo de contrato & $\begin{array}{l}1,069 * * * \\
(75,9 \%)\end{array}$ & $\begin{array}{l}1,256 * * * \\
(38,1 \%)\end{array}$ & $\begin{array}{l}0,940 * * * \\
(39,6 \%)\end{array}$ & $\begin{array}{l}1,506 * * * \\
(39 \%)\end{array}$ & $\begin{array}{l}1,145 * * * \\
(34,6 \%)\end{array}$ & $\begin{array}{l}1,182 * * * \\
(34,6 \%)\end{array}$ & $\begin{array}{l}1,251 * * * \\
(37,7 \%)\end{array}$ & $\begin{array}{l}1,101 * * * \\
(40,6 \%)\end{array}$ \\
\hline Atiguedad en empresa & $\begin{array}{l}0,791 * * \\
(56,2 \%)\end{array}$ & $\begin{array}{l}1,123 * * * \\
(34,1 \%)\end{array}$ & $\begin{array}{l}1,157 * * * \\
(48,8 \%)\end{array}$ & $\begin{array}{l}1,203 * * * \\
(31,1 \%)\end{array}$ & $\begin{array}{l}1,220 \text { *** } \\
(36,9 \%)\end{array}$ & $\begin{array}{l}1,064 * * * \\
(31,1 \%)\end{array}$ & $\begin{array}{l}1,181 \text { *** } \\
(35,6 \%)\end{array}$ & $\begin{array}{l}0,980 * * * \\
(36,1 \%)\end{array}$ \\
\hline Ocupación & $\begin{array}{l}0,371 * * \\
(26,4 \%)\end{array}$ & $\begin{array}{l}0,744 * * * \\
(22,6 \%)\end{array}$ & $\begin{array}{l}0,475 * * * \\
(20 \%)\end{array}$ & $\begin{array}{c}0,858 * * * \\
(22,2 \%)\end{array}$ & $\begin{array}{c}0,654 * * * \\
(19,8 \%)\end{array}$ & $\begin{array}{c}0,802 * * * \\
(23,5 \%)\end{array}$ & $\begin{array}{l}0,804 * * * \\
(24,2 \%)\end{array}$ & $\begin{array}{l}0,562 * * * \\
(20,7 \%)\end{array}$ \\
\hline CCAA & $\begin{array}{c}-0,113 * * \\
(-8 \%)\end{array}$ & $\begin{array}{l}0,054 * \\
(1,6 \%)\end{array}$ & $\begin{array}{l}-0,176 * * * \\
(-7,4 \%)\end{array}$ & $\begin{array}{l}0,126 * \\
(3,3 \%)\end{array}$ & $\begin{array}{l}0,092 \text { n.s. } \\
(2,8 \%)\end{array}$ & $\begin{array}{l}0,020 \text { n.s. } \\
(0,6 \%)\end{array}$ & $\begin{array}{l}-0,022 \text { n.s. } \\
(-0,7 \%)\end{array}$ & $\begin{array}{l}0,056 \text { n.s. } \\
(2,1 \%)\end{array}$ \\
\hline Estacionalidad & $\begin{array}{l}0,009 * * \\
(0,7 \%)\end{array}$ & $\begin{array}{l}0,006 \text { n.s. } \\
(0,2 \%)\end{array}$ & $\begin{array}{c}0,028 * \\
(1,2 \%)\end{array}$ & $\begin{array}{l}0,005 \text { n.s. } \\
(0,1 \%)\end{array}$ & $\begin{array}{l}0,008 \text { n.s. } \\
(0,2 \%)\end{array}$ & $\begin{array}{l}0,007 \text { n.s. } \\
(0,2 \%)\end{array}$ & $\begin{array}{l}0,008 \text { n.s. } \\
(0,2 \%)\end{array}$ & $\begin{array}{l}0,005 \text { n.s. } \\
(0,2 \%)\end{array}$ \\
\hline Parte no explicada & $\begin{array}{l}-0,783 \text { *** } \\
(-55,6 \%)\end{array}$ & $\begin{array}{l}-0,109 \text { n.s. } \\
(-3,3 \%)\end{array}$ & $\begin{array}{l}-0,144 \text { n.s. } \\
(-6,1 \%)\end{array}$ & $\begin{array}{l}0,143 \text { n.s. } \\
(3,7 \%)\end{array}$ & $\begin{array}{l}0,045 \text { n.s. } \\
(1,3 \%)\end{array}$ & $\begin{array}{l}0,016 \text { n.s. } \\
(0,5 \%)\end{array}$ & $\begin{array}{l}-0,189 \text { n.s. } \\
(-5,7 \%)\end{array}$ & $\begin{array}{l}-0,363 \text { n.s. } \\
(-13,4 \%)\end{array}$ \\
\hline Edad & $\begin{array}{l}0,440 \text { *** } \\
(31,3 \%)\end{array}$ & $\begin{array}{l}0,025 \text { n.s. } \\
(0,7 \%)\end{array}$ & $\begin{array}{l}-0,048 \text { n.s. } \\
(-2 \%)\end{array}$ & $\begin{array}{l}0,017 \text { n.s. } \\
(0,4 \%)\end{array}$ & $\begin{array}{l}0,028 \text { n.s. } \\
(0,9 \%)\end{array}$ & $\begin{array}{l}-0,003 \text { n.s. } \\
(-0,1 \%)\end{array}$ & $\begin{array}{l}0,203 \text { n.s. } \\
(6,1 \%)\end{array}$ & $\begin{array}{l}0,426 \text { n.s. } \\
(15,7 \%)\end{array}$ \\
\hline Formación & $\begin{array}{l}-0,096 * * * \\
(-6,8 \%)\end{array}$ & $\begin{array}{l}0,006 \text { n.s. } \\
(0,2 \%)\end{array}$ & $\begin{array}{l}0,008 \text { n.s. } \\
(0,3 \%)\end{array}$ & $\begin{array}{l}-0,010 \text { n.s. } \\
(-0,3 \%)\end{array}$ & $\begin{array}{l}0,001 \text { n.s. } \\
(0 \%)\end{array}$ & $\begin{array}{l}-0,002 \text { n.s. } \\
(-0,1 \%)\end{array}$ & $\begin{array}{l}0,018 \text { n.s. } \\
(0,6 \%)\end{array}$ & $\begin{array}{l}-0,008 \text { n.s. } \\
(-0,3 \%)\end{array}$ \\
\hline Estado civil & $\begin{array}{l}0,002 * * * \\
(0,2 \%)\end{array}$ & $\begin{array}{l}0,000 \text { n.s. } \\
(0 \%)\end{array}$ & $\begin{array}{l}0,000 \mathrm{n} . \mathrm{s} . \\
(0 \%)\end{array}$ & $\begin{array}{l}0,001 \text { n.s. } \\
(0 \%)\end{array}$ & $\begin{array}{l}0,000 \text { n.s. } \\
(0 \%)\end{array}$ & $\begin{array}{l}0,000 \text { n.s. } \\
(0 \%)\end{array}$ & $\begin{array}{l}0,001 \text { n.s. } \\
(0 \%)\end{array}$ & $\begin{array}{c}0,001 \text { n.s. } \\
(0 \%)\end{array}$ \\
\hline Sector actividad & $\begin{array}{l}-3,840 \text { n.s. } \\
(-272,7 \%)\end{array}$ & $\begin{array}{l}-0,173 \text { n.s. } \\
(-5,3 \%)\end{array}$ & $\begin{array}{l}-0,041 \text { n.s. } \\
(-1,7 \%)\end{array}$ & $\begin{array}{l}0,136 \text { n.s. } \\
(3,5 \%)\end{array}$ & $\begin{array}{l}-0,076 \text { n.s. } \\
(-2,3 \%)\end{array}$ & $\begin{array}{l}0,008 \text { n.s. } \\
(0,2 \%)\end{array}$ & $\begin{array}{l}-0,389 \text { n.s. } \\
(-11,7 \%)\end{array}$ & $\begin{array}{l}-0,138 \text { n.s. } \\
(-5,1 \%)\end{array}$ \\
\hline Tipo de contrato & $\begin{array}{l}0,135 \text { n.s. } \\
(9,6 \%)\end{array}$ & $\begin{array}{l}-0,042 \text { n.s. } \\
(-1,3 \%)\end{array}$ & $\begin{array}{l}-0,027 \text { n.s. } \\
(-1,2 \%)\end{array}$ & $\begin{array}{l}0,025 \text { n.s. } \\
(0,6 \%)\end{array}$ & $\begin{array}{l}0,026 \text { n.s. } \\
(0,8 \%)\end{array}$ & $\begin{array}{l}0,007 \text { n.s. } \\
(0,2 \%)\end{array}$ & $\begin{array}{l}-0,062 \text { n.s. } \\
(-1,9 \%)\end{array}$ & $\begin{array}{l}-0,291 \text { n.s. } \\
(-10,7 \%)\end{array}$ \\
\hline Atiguedad en empresa & $\begin{array}{l}0,957 \text { n.s. } \\
(68 \%)\end{array}$ & $\begin{array}{l}-0,007 \text { n.s. } \\
(-0,2 \%)\end{array}$ & $\begin{array}{l}0,023 \text { n.s. } \\
(1 \%)\end{array}$ & $\begin{array}{l}-0,013 \text { n.s. } \\
(-0,3 \%)\end{array}$ & $\begin{array}{c}-0,001 \text { n.s. } \\
(0 \%)\end{array}$ & $\begin{array}{l}0,000 \text { n.s. } \\
(0 \%)\end{array}$ & $\begin{array}{l}-0,040 \text { n.s. } \\
(-1,2 \%)\end{array}$ & $\begin{array}{l}0,020 \text { n.s. } \\
(0,8 \%)\end{array}$ \\
\hline Ocupación & $\begin{array}{c}1,744 * * * \\
(123,9 \%)\end{array}$ & $\begin{array}{l}0,022 \text { n.s. } \\
(0,7 \%)\end{array}$ & $\begin{array}{l}0,099 \text { n.s. } \\
(4,2 \%)\end{array}$ & $\begin{array}{l}-0,081 \text { n.s. } \\
(-2,1 \%)\end{array}$ & $\begin{array}{l}-0,030 \text { n.s. } \\
(-0,9 \%)\end{array}$ & $\begin{array}{l}0,006 \text { n.s. } \\
(0,2 \%)\end{array}$ & $\begin{array}{l}0,062 \text { n.s. } \\
(1,9 \%)\end{array}$ & $\begin{array}{l}0,075 \text { n.s. } \\
(2,8 \%)\end{array}$ \\
\hline CCAA & $\begin{array}{l}0,450 \text { n.s. } \\
(32 \%)\end{array}$ & $\begin{array}{l}0,006 \text { n.s. } \\
(0,2 \%)\end{array}$ & $\begin{array}{l}-0,039 \text { n.s. } \\
(-1,6 \%)\end{array}$ & $\begin{array}{l}0,053 \text { n.s. } \\
(1,4 \%)\end{array}$ & $\begin{array}{l}-0,020 \text { n.s. } \\
(-0,6 \%)\end{array}$ & $\begin{array}{l}0,003 \text { n.s. } \\
(0,1 \%)\end{array}$ & $\begin{array}{l}0,061 \text { n.s. } \\
(1,8 \%)\end{array}$ & $\begin{array}{l}0,198 \text { n.s. } \\
(7,3 \%)\end{array}$ \\
\hline Estacionalidad & $\begin{array}{l}0,354 \text { n.s. } \\
(25,1 \%)\end{array}$ & $\begin{array}{l}0,000 \text { n.s. } \\
(0 \%)\end{array}$ & $\begin{array}{l}0,000 \text { n.s. } \\
(0 \%)\end{array}$ & $\begin{array}{l}0,002 \text { n.s. } \\
(0,1 \%)\end{array}$ & $\begin{array}{l}0,000 \text { n.s. } \\
(0 \%)\end{array}$ & $\begin{array}{l}0,000 \text { n.s. } \\
(0 \%)\end{array}$ & $\begin{array}{l}0,000 \text { n.s. } \\
(0 \%)\end{array}$ & $\begin{array}{l}-0,002 \text { n.s. } \\
(-0,1 \%)\end{array}$ \\
\hline Constante & $\begin{array}{l}-0,929 \text { n.s. } \\
(-66 \%)\end{array}$ & $\begin{array}{l}0,054 \text { n.s. } \\
(1,7 \%)\end{array}$ & $\begin{array}{c}-0,120 \text { n.s. } \\
(-5 \%)\end{array}$ & $\begin{array}{l}0,013 \text { n.s. } \\
(0,3 \%)\end{array}$ & $\begin{array}{l}0,115 \text { n.s. } \\
(3,5 \%)\end{array}$ & $\begin{array}{l}-0,003 \text { n.s. } \\
(-0,1 \%)\end{array}$ & $\begin{array}{l}-0,044 \text { n.s. } \\
(-1,3 \%) \\
\end{array}$ & $\begin{array}{l}-0,644 \text { n.s. } \\
(-23,7 \%) \\
\end{array}$ \\
\hline
\end{tabular}

NOTA: Los valores entre paréntesis indican el peso de la contribución a la diferencia en la probabilidad de perder el empleo entre inmigrantes y nativos. $* * *, * *, *$ denota valores significativos al 1 por 100,5 por 100 y 10 por 100 respectivamente.

FUENTE: EPA (INE, 2005-2013). 
Los resultados obtenidos aplicando el método generalizado de descomposición de diferencias en el primer momento propuesto por Yun se muestran en los Cuadros 5,6 y 7, para la muestra total, la de hombres y la de mujeres respectivamente. La descomposición se realiza separadamente para el periodo de expansión y el de crisis. También se muestran los resultados obtenidos en el análisis detallado para cada uno de los años de recesión económica, lo que nos permite ver la evolución de los efectos detectados. En los cuadros se identifica la parte de la diferencia atribuible a cada variable, tanto en la parte explicada (diferencia en las características) como en la no explicada (diferencia en los coeficientes). Un signo negativo indica que la influencia de la variable, o de su parámetro, sobre la probabilidad de perder el empleo es favorable para los inmigrantes ya que contribuye reduciendo la diferencia entre las probabilidades de perder el empleo de inmigrantes y nativos. Entre paréntesis se indica el peso que cada contribución tiene en la diferencia.

La parte explicada (diferencia en las características), que mide como varía la probabilidad de perder el empleo en los extranjeros si tuvieran las mismas características que los nativos, es la que más aporta en la explicación (por encima del 80 por 100), sobre todo en el caso de las mujeres, y tanto en periodos de expansión como de crisis.

Las variables que a su vez más aportan a la explicación de esta diferencia son el tener un contrato temporal y con poca antigüedad en la empresa, tanto para hombres como para mujeres, segmentos laborales ambos donde la concentración de los trabajadores extranjeros es muy superior a la de los nativos. Como se observa en el Cuadro 1, casi la mitad de los trabajadores inmigrantes tienen un contrato temporal, mientras que esta cifra se sitúa en el 25 por 100 para el caso de los nativos. En relación a la antigüedad, si bien el 67 por 100 de los extranjeros llevan menos de 3 años en la empresa, el dato es casi la mitad (36 por 100) si nos referimos a los trabajadores nativos.

A su vez se observa que la aportación de ambas variables, que se sitúa en torno a 1 punto porcentual durante el periodo de expansión, tanto para hombres como para mujeres, incrementa durante el periodo de crisis, sobre todo en el caso de los hombres. En concreto, para este colectivo, la aportación del tipo de contrato casi se triplica, hasta los 2,8 puntos porcentuales, y la de la antigüedad casi se duplica, hasta los 1,8 puntos porcentuales. Todo ello indica que la principal causa de que la probabilidad de perder el empleo se haya incrementado más entre los trabajadores inmigrantes que entre los nativos durante el periodo de crisis, se debe a que los extranjeros tienen, en mayor medida que los nativos, contratos con menores costes de despido, es decir, contratos temporales y con poca antigüedad en la empresa.

Otras variables con una elevada aportación a la explicación de la diferencia en las probabilidades de perder el empleo entre inmigrantes y extranjeros es el sector de actividad, en la muestra de hombres, y la ocupación en la de mujeres. En ambos casos dicha aportación incrementa durante el periodo de crisis respecto a los años de expansión. En concreto, la aportación del sector de actividad en el caso de los hombres pasa de no aportar prácticamente nada a la diferencia durante el periodo 
de expansión a aportar casi 1 punto porcentual durante la crisis. Este dato refleja que la mayor concentración de los inmigrantes en el sector de la construcción y el agrario, los más castigados por la crisis, es otra de las principales razones que justifican el mayor incremento en la probabilidad de perder el empleo en los hombres inmigrantes frente a los nativos durante el periodo de recesión. En el caso de las mujeres, la aportación de la variable ocupación pasa de 0,4 puntos porcentuales en el periodo de expansión a 0,7 puntos porcentuales en la crisis, lo que se explica por la mayor concentración de las mujeres inmigrantes en las ocupaciones de baja cualificación que, a su vez, es donde más ha incrementado la probabilidad de perder el empleo durante la etapa de recesión económica.

En relación a la aportación de la parte no explicada (diferencia en los coeficientes), que mide como varía la probabilidad de perder el empleo en los extranjeros cuando se les aplica la percepción que el mercado tiene para los nativos (es decir, los parámetros estimados para los nativos), tiene una contribución más residual a la diferencia en la probabilidad de perder el empleo entre inmigrantes y extranjeros, aunque con un comportamiento distinto según el género.

En la muestra de mujeres, mientras que la contribución de la parte no explicada no resultó estadísticamente significativa durante el periodo de crisis, si lo fue durante el ciclo expansivo pero con signo negativo. Estos resultados, similares a los obtenidos en el análisis de la modelización logit, indican la existencia de una discriminación positiva a favor de la inmigrante en los periodos de expansión, justificada en parte por la extranjerización del sector doméstico comentada con anterioridad. De hecho el sector de actividad es la variable que más peso tiene en la contribución a la explicación de la diferencia en la probabilidad de perder el empleo reduciendo la misma en casi 4 puntos porcentuales.

Sin embargo, en la muestra de hombres se obtuvo una aportación positiva y estadísticamente significativa tanto en periodos de expansión (medio punto porcentual) como de crisis (1,3 puntos porcentuales), indicando la existencia de algún tipo de discriminación negativa contra el inmigrante en ambos periodos. El componente que más aporta a la explicación de la diferencia es el parámetro del término independiente, el cuál recoge el efecto de factores no observables no incluidos en el modelo, que podrían estar relacionados con diferencias en la productividad laboral asociadas a la calidad educativa recibida en los países de origen. A su vez, se observa una tendencia creciente durante los últimos años de la recesión económica, incrementándose su aportación hasta casi 2 puntos porcentuales en el año 2013.

Por último, merece la pena destacar, que en la muestra de hombres y para el caso de la parte no explicada, la aportación de las variables tipo de contrato y antigüedad en la empresa cambia de signo según el ciclo económico, lo que muestra indicios de discriminación negativa contra el inmigrante en el tratamiento de estas variables durante el periodo de crisis. Si bien las aportaciones no resultan muy elevadas si merece la pena destacar que se observa una tendencia creciente en ambos casos a medida que avanza la crisis. Todo ello pone de manifiesto un incremento en las dificultades que encuentran los inmigrantes para estabilizarse en sus puestos de trabajo. 


\section{Conclusiones}

El impacto que la crisis económica ha tenido en el mercado laboral ha sido de mayor intensidad en los inmigrantes que en los nativos, incrementándose, más del doble, las diferencias registradas en la probabilidad de perder el empleo entre ambos colectivos durante el periodo de recesión. En este contexto, el objetivo de esta investigación es aportar evidencia empírica sobre si esta tendencia se explica por la existencia de diferentes características laborales entre nativos e inmigrantes, debido a la mayor concentración de estos últimos en los segmentos más castigados por la crisis, o si existen otros factores inobservables que indiquen, a igualdad de condiciones, mayor frecuencia en la pérdida de empleo del inmigrante que en la del nativo.

Los resultados obtenidos permiten afirmar que la mayor parte de la diferencia en la probabilidad de perder el empleo entre inmigrantes y nativos (más del 80 por 100) se justifica, tanto en periodos de expansión como de crisis, por las distintas características laborales de ambos colectivos, siendo las variables en las que se detectaron mayores diferencias, y por tanto que más aportan a la explicación de la brecha, el tipo de contrato y la antigüedad en la empresa.

A su vez, la contribución de ambas variables a la explicación de la diferencia ha incrementado en el periodo de crisis respecto al de expansión, casi triplicándose en el caso del tipo de contrato y casi duplicándose para la variable antigüedad. Con todo, la principal causa que explica el incremento en la brecha durante el periodo de recesión es el hecho de que los inmigrantes se concentran, en mayor medida que los nativos, en los segmentos laborales donde los costes de despido son menores. Así, mientras que casi el 50 por 100 de los trabajadores inmigrantes tiene un contrato temporal y el 67 por 100 de ellos lleva menos de tres años en la empresa, las cifras se reducen a casi la mitad en el caso de los nativos (el 25 por 100 de ellos tienen un contrato temporal y el 36 por 100 lleva menos de tres años en la empresa).

Tan sólo se detecta la existencia de discriminación negativa, aunque de baja intensidad, en la muestra de hombres, al obtenerse diferencias estadísticamente significativas en los parámetros de las variables cuando la estimación se realiza diferenciando por nacionalidad. En concreto, la valoración que hace el mercado del tipo de contrato y de la antigüedad en la empresa no es la misma según se trate de un trabajador nativo o inmigrante. La existencia de factores no incluidos en el modelo, como el hecho de que las diferencias en la calidad educativa recibida en el país de origen podrían generar distintos niveles de productividad, podrían explicar en parte los resultados obtenidos. Si bien la cuantía de este componente discriminatorio no resulta elevada, si merece la pena destacar que se observa una tendencia creciente en la misma durante el periodo de recesión, lo que pone de manifiesto un incremento en las dificultades que encuentran los inmigrantes para estabilizar sus puestos de trabajo.

Con todo, la investigación aporta evidencia sobre el freno que han sufrido los procesos de asimilación de la población inmigrante en el mercado laboral español 
durante el periodo de crisis. En este sentido es necesario, por parte de las autoridades gubernamentales, el desarrollo de políticas sociales y económicas que reduzcan las prácticas discriminatorias en los procesos de estabilización laboral. Conseguir la integración de los inmigrantes en el mercado laboral, generará beneficios tanto desde un punto de vista social como económico, en la medida en que la aportación del inmigrante a la economía nacional estará vinculada a su situación en el mercado laboral.

\section{Referencias bibliográficas}

[1] AMUEDO DORANTES, C. y DE LA RICA, S. (2013): «The immigration surplus and the substitutability of immigrant and native labor: evidence from Spain». Empirical Economics, 44(2), 945-958.

[2] AMUEDO DORANTES C. y DE LA RICA, S. (2007): «Labour Market Assimilation of Recet Immigrants in Spain», British Journal of Industrial Relations, 45(2), 257-284.

[3] BEVELANDER P. y NIELSEN, H. S. (2001): «Declining employment success of immigrant males in Sweden: observed or unobserved characteristics», Journal of Population Economics, 14(3), 455-472.

[4] BLINDER, A. S. (1973): «Wage discrimination: reduced form and structural variables», Journal of Human Resources, 8 (4), 436-455.

[5] BORJAS, G. (1999): Heaven's Door: Immigration Policy and the American Economy. Princenton University Press.

[6] BORJAS, G.; FREEMAN, R. y KATZ, L. (1997): «How much do Immigration and Trade Affect Labor Market Outcomes», Bookings Papers on Economic Activity, 1:1-90.

[7] BORJAS, G. J. (1995): «Assimilation and changes in cohort quality revisited:what happened to immigrant earnings in the 1980s?», Journal of Labor Economics, 13 (2), 201-245.

[8] CANAL DOMÍNGUEZ, J. F. y RODRÍGUEZ GUTIÉRREZ, C. (2008): «Analysis of wage differences between native and immigrant workers in Spain», Spanish Economic Review, 10, 109-134.

[9] CAPARRÓS, A. y NAVARRO, L. (2010): «Determinantes de la contratación indefinida en España: nacionales versus extranjeros», Revista de Economía Laboral, 7, 39-63.

[10] CARD, D. (2005): «Is new immigration really so bad?», The Economic Journal, 115, 300-323.

[11] CARRASCO, R.; JIMENO, J. F. y ORTEGA, A. C. (2008): «The effect of immigration on the labor market performance of native-born workers: some evidence for Sapin», Journal of Population Economics, 21 (3): 627-648.

[12] CHISWICK, B. R. (1978): «The effects of Americanization on the earnings of foreignborn men», Journal of Political Economy, 86(5), 897-922.

[13] CHISWICK, B.; COHEN, Y.y ZACH, T. (1997): «The labor market status of immigrants: effects of unemployment rate at arrival and duration of residence», Industrial \& Labor Relations Review, 50(2), 289-303.

[14] CLARK, K. and LINDLEY, J. (2005): «Immigrant Labour Market Assimilation and Arrival Effects: Evidence from the Labour Force Survey», Sheffield Economic Research Paper Series, No. 2005004, University of Sheffield. 
[15] CONSTANT, A. y MASSEY, D. S. (2003): «Self-selection, earnings and out- migration: a longitudinal study of immigrants to Germany», Journal of Population Economics, 16 (4), 631-653.

[16] DE NEW, J. P. y ZIMMERMANN, K. (1994): «Native Wage Impacts of Foreign Labor: A Random Effects Panel Analysis», Journal of Population Economics, 7 (2): 177-192.

[17] DOLADO, J. J.; JIMENO, J. F. y DUCE, R. (1997): «Los efectos de la inmigración sobre la demanda relativa de trabajo cualificado vs poco cualificado: evidencia para España», Cuadernos Económicos de ICE, 63: 11-29.

[18] DULEEP, H. O. y DOWHAN, D. J. (2002): «Insights from longitudinal data on earnings of US foreign born men», Demography, 39, 485-506.

[19] FERNÁNDEZ, C. y ORTEGA, C. (2008): «Labor market assimilation of immigrants in Sapin: employment at the expense of bad job-matches?», Spanish Economic Review, 10, 83-107.

[20] FRIEDBERG, R (2000): «You can't take it with you? Immigrant assimilation and the portability of human capital: evidence from Israel», Journal of Labor Economics, 18 (2), 221-251.

[21] FRIEDBERG, R. y HUNT, J. (1995): «The Impact of Immigration on Host Country Wages, Employment and Growth», Journal of Economic Perspectives, 9 (2), 23-44.

[22] GARCÍA-SERRANO, C.; ARRANZ, J. M.; CANTÓ, O. y MALO, M. A. (2012): El sistema español de protección y la duración efectiva de los episodios de paro, Premio Fipros 2011/02, Ministerio de Empleo y Seguridad Social.

[23] GARDEAZÁBAL J. y UGIDOS, A. (2004): «More on identification in detailed wage descompositions», The Review of Economics and Statistics, 86, 1034-1036.

[24] GREENWOOD, M. y McdOWELL, J. (1986): «The Factor Market Consequences of U.S. Immigration», Journal of Economic Literature, 24 (4), 1738-1772.

[25] HERRARTE, A.; MEDINA, E. y VICÉNS, J. (2007): «Cambios en la situación laboral de la población española ante el incremento de la inmigración», Ekonomiaz, Revista Vasca de Economía, 66, 330-349.

[26] MARTÍNEZ, R. (2011): «La reorganización de los cuidados familiares en un contexto de inmigración internacional», Cuadernos de Relaciones Laborales, 29 (1), 93-123.

[27] MEDINA, E.; HERRARTE, A. y VICÉNS, J. (2010): «Inmigración y desempleo en España: impacto de la crisis económica», Revista de Información Comercial Española, 854, 37-48.

[28] OAXACA, R. (1973): «Male-female wage differentials in urban labor markets», International Economic Review, 14 (3), 693-703.

[29] PISCHKE J. y VELLING, J. (1997): «Employment Effects of Immigration to Germany: an Analysis Based on Local Labor Markets», Review of Economics and Statistics, 79 (4), 594-604.

[30] SIMÓN, H.; SANROMÁ, E. y RAMOS, R. (2008): «Labour segregation and immigrant and native-born wage distribution in Sapin: an anlysis using matched employer-employee data», Spanish Economic Review, 10, 135-168.

[31] WHEATLY, P. S. (1999): «The employment adjustment of male immigrants in England», Journal of Population Economic, 14 (1), 193-220.

[32] YUN, M. (2004): «Descomposing differences in the first moment», Economic Letter, 82 (2), 275-280. 